\title{
A brain-specific isoform of mitochondrial apoptosis-inducing factor: AIF2
}

\author{
E Hangen ${ }^{1,2,3}$, D De Zio ${ }^{4,5}$, M Bordi $^{4,5}$, C Zhu ${ }^{6}$, P Dessen, ${ }^{2,3,7}$, F Caffin ${ }^{1,2,3}$, S Lachkar ${ }^{1,2,3}$, J-L Perfettini ${ }^{1,2,3}$, V Lazar, ${ }^{2,3,8}$, J Benard ${ }^{2,3,9}$, \\ GM Fimia $^{10}$, M Piacentini ${ }^{10}$, F Harper ${ }^{11}$, G Pierron ${ }^{11}$, JM Vicencio ${ }^{1,2,3}$, P Bénit ${ }^{12,13,14}$, A de Andrade $^{15}$, G Höglinger ${ }^{15}, C_{\text {Culmsee }}{ }^{16}$, \\ P Rustin ${ }^{12,13,14}$, K Blomgren $^{6,17}$, F Cecconi ${ }^{4,5}$, G Kroemer $^{\star, 1,2,3,18}$ and N Modjtahedi ${ }^{\star, 1,2,3,18}$
}

Apoptosis-inducing factor (AIF) has important supportive as well as potentially lethal roles in neurons. Under normal physiological conditions, AIF is a vital redox-active mitochondrial enzyme, whereas in pathological situations, it translocates from mitochondria to the nuclei of injured neurons and mediates apoptotic chromatin condensation and cell death. In this study, we reveal the existence of a brain-specific isoform of AIF, AIF2, whose expression increases as neuronal precursor cells differentiate. AIF2 arises from the utilization of the alternative exon $2 b$, yet uses the same remaining 15 exons as the ubiquitous AIF1 isoform. AIF1 and AIF2 are similarly imported to mitochondria in which they anchor to the inner membrane facing the intermembrane space. However, the mitochondrial inner membrane sorting signal encoded in the exon $2 b$ of AIF2 is more hydrophobic than that of AIF1, indicating a stronger membrane anchorage of AIF2 than AIF1. AIF2 is more difficult to be desorbed from mitochondria than AIF1 on exposure to non-ionic detergents or basic pH. Furthermore, AIF2 dimerizes with AIF1, thereby preventing its release from mitochondria. Conversely, it is conceivable that a neuron-specific AIF isoform, AIF2, may have been 'designed' to be retained in mitochondria and to minimize its potential neurotoxic activity.

Cell Death and Differentiation (2010) 17, 1155-1166; doi:10.1038/cdd.2009.211; published online 29 January 2010

Apoptosis-inducing factor (AIF) has initially been described as a mitochondrial intermembrane protein that is released from mitochondria under conditions of cell death induction and that can induce isolated nuclei to undergo nuclear shrinkage and chromatinolysis, two features that are classically associated with apoptosis. ${ }^{1}$ Since its discovery 10 years ago, the AIF protein has been characterized at the structural level, ${ }^{2,3}$ and the AIF gene has been subjected to genetic manipulations in mice, flies, nematodes and yeast, revealing the phylogenetically conserved contribution of AIF to cell death in multiple systems. ${ }^{4,5}$

After the mitochondrial import of the precursor AIF protein and the removal of its $\mathrm{N}$-terminal 53 amino acids, which includes a mitochondrial localization sequence (MLS), the processed mature human AIF $62 \mathrm{kDa}$ is inserted into the inner mitochondrial membrane, with the $\mathrm{N}$-terminus facing the matrix and with the $\mathrm{C}$-terminal catalytic domain exposed to the intermembrane space. ${ }^{6}$ The mitochondrial AIF protein is an $\mathrm{NAD}(\mathrm{P}) \mathrm{H}$ oxidase $^{7}$ whose local redox function is essential for optimal oxidative phosphorylation. ${ }^{8}$ Knockdown, deletion or hypomorphic mutation of $A I F$ (the harlequin or $\mathrm{Hq}$ mutation) reduces the expression of complex I subunits in the respiratory chain, ${ }^{8}$ thereby provoking a mitochondriopathy that leads to progressive neurodegeneration, photoreceptor loss and cardiomyopathy. ${ }^{9-13}$ The consistent finding that the targeting of AlF mostly affects the central nervous system $(\mathrm{CNS})^{9}$ might either be explained by the general tendency of complex I mitochondriopathies to manifest at the level of the $\mathrm{CNS}^{14}$ and/or by an implication of AIF in the differentiation of neuronal cell precursors. ${ }^{12}$

On apoptotic stimuli, AIF, which is able to directly interact with DNA, ${ }^{15}$ translocates to the nucleus and participates in chromatin condensation and chromatinolysis. ${ }^{16}$ The switch from the vital to the lethal functions of AIF is spatially regulated by its subcellular localization and tightly controlled by at least two processes: (i) outer mitochondrial membrane permeabilization (MOMP), which is regulated by multiple mitochondrial proteins including members of the Bcl-2 family, ${ }^{17}$ (ii) activation of a series of non-caspase cysteine proteases (including calpains and cathepsins) that cleave the $\mathrm{N}$-terminal membrane

\footnotetext{
${ }^{1}$ INSERM U848, Villejuif, France; ${ }^{2}$ Institut Gustave Roussy, Villejuif, France; ${ }^{3}$ University Paris 11, Orsay, France; ${ }^{4}$ Department of Biology, Dulbecco Telethon Institute, University of Rome, 'Tor Vergata', Rome, Italy; ${ }^{5}$ Laboratory of Molecular Neuroembryology, IRCCS Fondazione Santa Lucia, Rome, Italy; ${ }^{6}$ Center for Brain Repair and Rehabilitation, Institute of Neuroscience and Physiology, University of Gothenburg, Gothenburg, Sweden; ${ }^{7}$ CNRS FRE2939, Vilejuif, France; ${ }^{8}$ Functional Genomic Unit, Villejuif, France; ${ }^{9} \mathrm{CNRS}$ UMR8126, Villejuif, France; ${ }^{10}$ National Institute for Infectious Diseases, 'Lazzaro Spallanzani', Rome, Italy; ${ }^{11}$ Laboratoire 'Réplication de I'ADN et Ultrastructure du Noyau' CNRS-Institut André Lwoff, Villejuif 94801, France; ${ }^{12}$ INSERM U676, Paris, France; ${ }^{13}$ Hôpital Robert Debré, Paris, France; ${ }^{14}$ University René Diderot, Paris, France; ${ }^{15}$ Experimental Neurology, Philipps University, Marburg, Germany; ${ }^{16}$ Department of Pharmacy, Institute for Pharmacology and Toxicology, Philipps-University Marburg, Marburg, Germany and ${ }^{17}$ Department of Pediatric Oncology, Queen Silvia Children's Hospital, Gothenburg, Sweden

*Corresponding authors: G Kroemer, INSERM, U848, Institut Gustave Roussy, Pavillon de Recherche 1, F-94805 Villejuif, Paris, France. Tel: + 33142116046 ; Fax: + 331421160 47; E-mail: kroemer@ orange.fr or N Modjtahedi, Tel: + 3314211 54 91; Fax: + 331421166 65; E-mail: nazanine@igr.fr

${ }^{18}$ These authors share co-senior authorship.

Keywords: brain development; neural differentiation; neural progenitor; neuroblastoma; oxidative phosphorylation

Abbreviations: CNS, central nervous sytem; FISH, fluorescent in situ hybridization; LUHMES, Lund Human Mesencephalic neurons; IMSS, inner membrane sorting signal; MLS, mitochondrial localization signal; MOMP, mitochondrial outer membrane permeabilization. NCI, National Cancer Institute; NLS, nuclear localization signal; TEM, transmission electron microscopy

Received 01.7.09; revised 11.11.09; accepted 01.12.09; Edited by JC Martinou; published online 29.1.10
} 
insertion domain at amino acid 101, thus catalyzing the de-attachment of mature AIF from the inner mitochondrial membrane. ${ }^{5,6,18}$ The nuclear translocation of AIF could be inhibited by the overexpression of heat shock protein 70 , which can intercept AIF in the cytosol ${ }^{19}$ or deletion of cyclophilin $A$, which is required for AIF to move into the nucleus. ${ }^{20}$ In mice, the $H q$ mutation has been shown to reduce acute neuronal cell death after ischemia, hypoglycemia and neurotrauma in young animals, before they manifest the $\mathrm{Hq}$ associated neurodegeneration. ${ }^{21}$ However, the $\mathrm{Hq}$ mutation had no cardioprotective effect ${ }^{13}$ and was not able to make islet beta cells more resistant to hydrogen peroxide-induced cell death, ${ }^{22}$ suggesting that AIF contributes to lethal signaling in a cell type-specific manner.

Through alternative splicing, the precursor mRNA transcribed from the AIF gene can give rise to several distinct proteins. Thus, alternative utilization of the exons $2 a$ or $2 b$ of the AIF gene (16 exons in total) gives rise to AIF1 (the originally described isoform of AIF) or AIF2 (which has been found in cDNA libraries from fetal mouse tissues), respectively. AIF1 and AIF2 only differ in a short stretch of their amino acid sequence in the $\mathrm{N}$-terminal region that is removed from the mature protein as it translocates to the nucleus. ${ }^{23}$ Numerous functional studies have been performed on AIF1, the most abundant and ubiquitous AIF isoform, whereas, AIF2 has not been further characterized. In addition to the above mentioned isoforms, an alternate transcriptional start site located at intron 9 of AIF originates a short variant of the protein (AIFsh) that lacks the N-terminal MLS and the redoxactive domain, yet retains the nuclear localization sequence (NLS). The transfection-enforced overexpression of AIFsh results in a nuclear protein that causes apoptosis. ${ }^{24}$ Moreover, another short form of AIF, AIFsh2, results from the alternative utilization of exon $9 \mathrm{~b}$ (instead of 9 ), which contains a stop codon. AlFsh2 is a truncated protein that lacks the C-terminal pro-apoptotic domain, yet conserves its mitochondrial localization and redox function. ${ }^{25}$ Both short AIF isoforms appear to be low-abundant in normal tissues because they have not been detected by immunoblot, with the exception of AIFsh2 that reportedly is present in the liver extracts. ${ }^{24,25}$

By characterizing the tissue expression profile of AIF isoforms, we discovered that AIF2 is specifically expressed in the CNS. Driven by the pathophysiological effect of AIF in neurodegeneration, ${ }^{9,11,12}$ we performed an exhaustive functional and biochemical characterization of the AIF2 isoform and importantly, we found that, compared with AIF1, AIF2 possess a stronger anchoring capacity to the inner mitochondrial membrane. These results suggest that the neuronspecific AIF2 isoform has been 'designed' for maintaining its mitochondrial functions yet reducing its pro-apoptotic activities.

\section{Results and Discussion}

A novel brain-specific isoform of AIF, AIF2. When sequencing several cDNAs from fetal human brain, we detected an alternative exon 2 usage (Figure 1a), indicating that the precursor of the human AIF mRNA can be alternatively spliced, yielding two isoforms that we designated AIF1 (when exon $2 a$ is used) and AIF2 (when exon $2 b$ is used). The alternative exons $2 a$ and $2 b$ are phylogenetically conserved among mammals. Similarly, the two exons were generated by gene duplication well before the speciation of mammals (data not shown). Indeed, we were able to trace the duplication event to chicken genome, in which a region homologous to the mammalian exon 2 a can be found $5^{\prime}$ of exon 2 b on chromosome 4 , although the splice acceptor site of exon 2a seems to be non-canonical (UCSC, genome browser). Thus, both exons $2 \mathrm{a}$ and $2 \mathrm{~b}$ are detectable in published cDNA sequences from primates, rodents and other mammals (such as Equus caballus and Canis familiaris) (NCBI-Homologen database), whereas only one exon 2 , which resembled exon $2 \mathrm{~b}$ from mammals, could be discerned in chicken cDNA libraries (Figures $1 \mathrm{~b}$ and $\mathrm{c}$ ). Amino acid alignments of exons $2 a$ and $2 b$ from several animal species revealed two positively charged and one negatively charged common residues, as well as conserved motifs with hydrophobic stretches (Figure 1c). However, secondary structure predictions indicate the consequences of variable residues and their differential properties on the potential secondary structure of the segment encoded by each of the two exons (Figure 1d).

Quantitative expression profiling of the two AIF isoforms have shown that AIF2 mRNA was specifically detected in human brain, yet was absent from most other analyzed tissues except the retina (Figure $2 a$ ). Within the human adult brain, AIF2 mRNA was found in all regions, including the cortex or in subcortical areas, and the expression level of $A / F 1$ and AIF2 mRNAs were similar (Figure 2b). This general expression profile was similar for all examined mouse tissues, in which AIF2 mRNA was again restricted to the brain (Figure 2c). Both in human and mice, low levels of AIF expression were detected in the brain compared with other organs. This probably reflects the comparative paucity of mitochondria in the brain, because the ratio of AIF protein and the most abundant outer mitochondrial membrane protein, VDAC, is similar in a panel of distinct mouse organs including brain. ${ }^{14}$ The expression levels of AIF1- and AIF2-specific mRNAs were equivalent in the adult mouse brain and were similarly affected by the hypomorphic $\mathrm{Hq}$ mutation that reduced the expression of both $A I F 1$ and $A I F 2$ to around $20 \%$ of the control level (Figure $2 \mathrm{~d}$ ). It is noteworthy that the expression of human AIF2 mRNA was higher in adult brain than in fetal brain (while that of AIF1 was lower) (Figure 2b), indicating that the AIF1/AIF2 ratio decreases as brain cells differentiate. Accordingly, mouse embryonic telencephalic cells immortalized with thermosensitive SV40 large T antigen (tsA58 LT-Ag) (Figures $3 a$ and $b$ ), or human embryonic mesencephalic cells immortalized with a v-Myc retroviral vector (Figure $3 \mathrm{c}$ ) could be stimulated to express higher AIF2 levels on in vitro differentiation. Thus, AIF2 is specifically and differentially expressed in brain cells, depending on their maturation status.

Next, we determined the relative AIF1 and AIF2 mRNA expression in the $\mathrm{NCl}$ (National Cancer Institute) panel of cancer cell lines (NCI60). Although AIF1 was expressed by all cells, independently of their tissue origin, AlF2 was absent from all samples (Supplementary Figure 1), including from brain cancer cell lines, of glial origin, contained in the $\mathrm{NCl}$ 


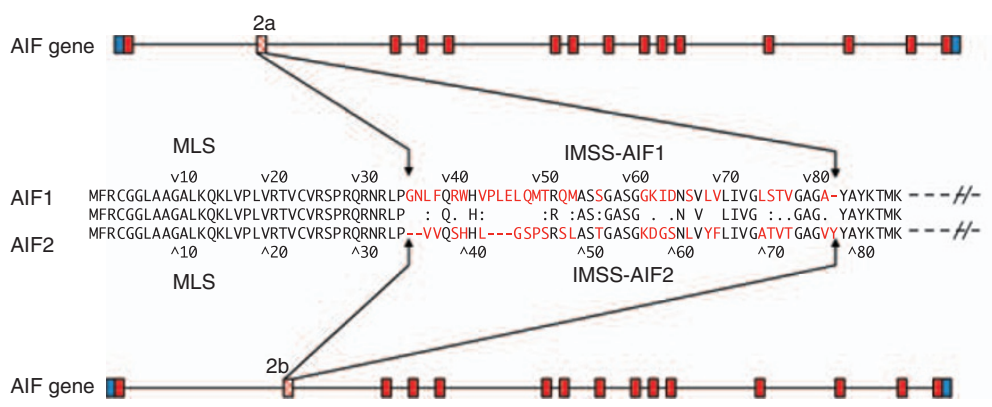

C

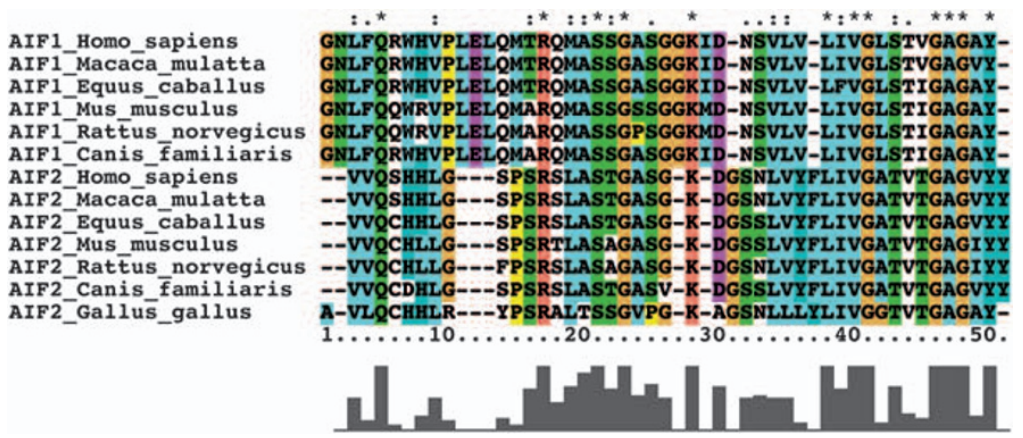

b

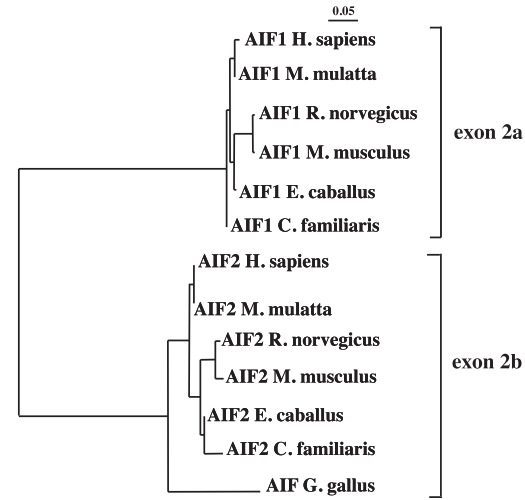

d

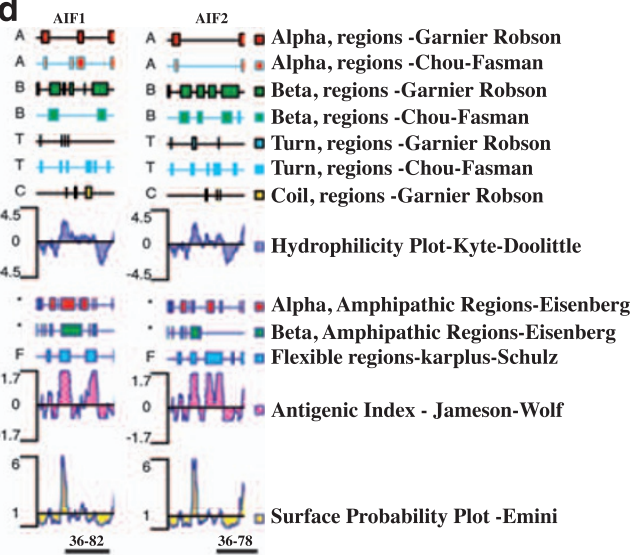

Figure 1 Comparison of AIF1 and AIF2 sequences. (a) AIF1 and AIF2 are alternative splice variants transcribed from AlF locus. Alternative usage of exon 2a or 2b of AIF gene allows the synthesis of respectively AIF1 and AIF2 isoforms. Human AIF1 and AIF2 share an identical N-terminal (aa 1-35) mitochondrial localization signal (MLS) but bear different inner membrane sorting signal (IMSS-AIF1: aa 35-82 and IMSS-AIF2: aa 35-78) encoded respectively by exon 2a and 2b. The rest of the protein is identical for both isoforms. Primary amino acid sequence comparison for MLS and IMSS regions of AIF1 and AIF2 were achieved using DNASTAR multiple alignment program. Residues differing between the two isoforms are colored in red. (b) Phylogenetic analyses of AIF1 and AIF2. Mutiple alignment of exon 2-encoded polypeptides, available from public databases, reveals a restricted simultaneous usage of exon $2 \mathrm{a}$ and $2 \mathrm{~b}$ to mammals. Chicken AIF protein is rooted to mammalian AIF2. This tree is obtained using neighbor joining method after clustalW multiple alignment. Refseq identifiers for analyzed protein sequences are the followings: Homo sapiens AIF1 (NP_004199), AIF2 (NP_665811); Macaca mulata AIF1 (XP_001092146), AlF2 (XP_001092025); Equus Caballus AIF1 (XP_001915191), AlF2 (XP_001915198); Canis familiaris AlF1 (XP_538170), (AlF2 XP_865808); Mus musculus AIF1 (NP_036149), AIF2 (CAM22220); Rattus norvegicus AIF1 (NP_112646), AIF2 (EDM10916); Gallus gallus AIF (NP_001007491). (c) AIF1IMSS and AIF2-IMSS share conserved amino acids. Multiple alignment of exon 2a-encoded against exon $2 b$-encoded polypeptide segments, from the same organisms presented in $1 \mathrm{~b}$, was achieved using clustalW multiple alignment program. Star indicates fully conserved residues. Dots indicate residues with similar properties. The histogram of residue conservation is presented at the bottom of the figure. (d) Prediction of secondary structural characteristics and physicochemical properties of the $\mathrm{N}$-terminal segments of AIF1 and AIF2. Entire primary amino acid sequences of AIF1 and AIF2 were analyzed using DNASTAR Protean Secondary structure prediction program and only the result for the N-terminal segment (MLS + IMSS) of each isoform is shown

panel (Figure 4a). However, AIF2 was expressed by a fraction of neuroblastomas (Figures $4 a$ and $b$ ), in line with the data obtained on normal, untransformed tissues (see above, Figure 2). In conclusion, AIF2 is specific for neuronal tissues and neuroblastomas.

Cellular and subcellular localization of AIF2. Comparative in situ hybridizations of the adult mouse brain with isoformspecific probes revealed a similar macroscopic distribution of AIF1 and AIF2 mRNAs, with peak intensities in the olfactory bulb, rostral migratory stream, olfactory cortex and pituitary (Figures 5a-f, negative controls with anti-sense probes are shown in Supplementary Figure 2). To analyze whether both $A I F$ isoforms are expressed in the same cells, we performed a simultaneous fluorescent in situ hybridization (FISH) with differentially labeled exon $2 \mathrm{a}$ - and $2 \mathrm{~b}$-specific probes (red and green, respectively). In most brain regions, $A / F 1$ and AIF2 were co-expressed by the same cells. However, approximately $25 \%$ of the cells present in the anterior olfactory nucleus stained uniquely for the exon $2 \mathrm{~b}$-specific probe, indicating the existence of brain cells that solely express $A I F 2$ (and not $A I F 1$ ) (Figures $5 \mathrm{~g}-\mathrm{i}$ ).

AIF1 and AIF2 share an identical N-terminal MLS, while their exon 2-encoded inner membrane sorting signals (IMSS) differ (Figure 1). To analyze whether this difference might affect the mitochondrial localization of AIF2, we compared the subcellular distribution of AIF1 and AIF2 by transfecting Hela cells with Flag-tagged versions of AIF1 or AIF2 (with the Flag fused to the C-terminus). When transfected cells were fixed and permeabilized with paraformaldehyde plus Triton X100 and stained with a Flag-specific antibody (Figure 6a), we found both proteins similarly redistributed into mitochondria 
a

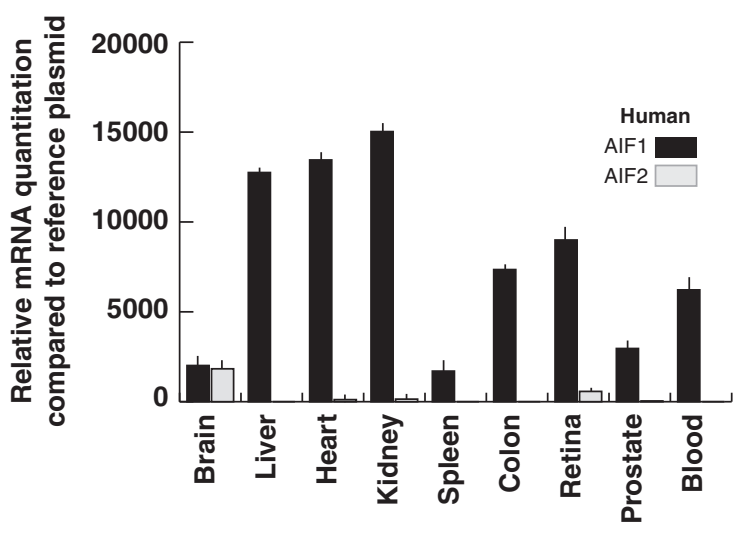

C

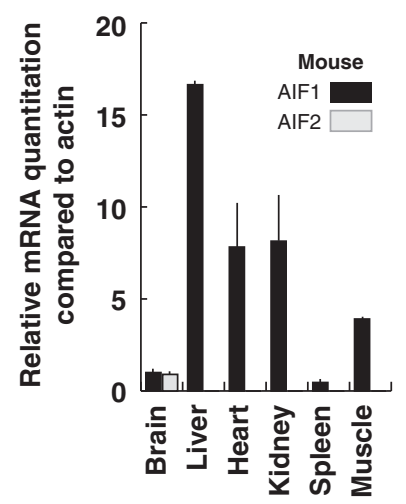

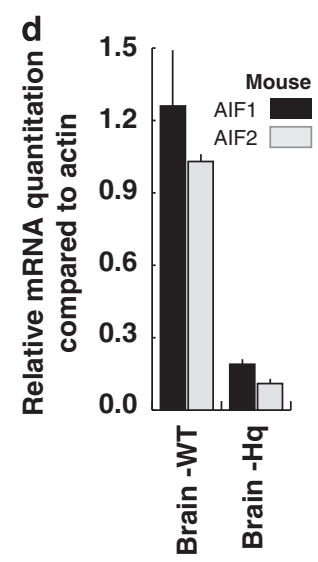

Figure 2 AlF2 mRNA expression is restricted to the brain. Quantitative expression analyses of AIF1 and AIF2 mRNAs in various human (a) and murine (c) organs or in various human brain sub-regions (b) were performed. AIF1 and AIF2 mRNA expression was compared between brains of wild-type (wt) and hypomorphic Harlequin $(\mathrm{Hq})$ mutant mice $(\mathbf{d})$. Data obtained for human samples $(\mathbf{a}, \mathbf{b})$ correspond to three independent quantifications (mean \pm S.D.) and for mouse organs $(\mathbf{c}, \mathbf{d})$ represent means \pm S.D. from at least three independent experiments. Relative quantification of AlF1 and AIF2 mRNA expression levels in human samples $(\mathbf{a}, \mathbf{b})$ was achieved by standard curve method using an AIF1/AIF2 reference plasmid. For murine RNA samples, relative fold variation of AIF1 and AIF2 were calculated using the comparative $C_{\mathrm{t}}$ method (which were labeled with a matrix-targeted fluorescent protein, dsRed-mito), with no discernible effects on the overall shape of the mitochondrial network. Differential permeabilization of the outer mitochondrial membrane (with $0.4 \mathrm{mg} / \mathrm{ml}$ digitonin) and the inner membrane (with $0.8 \mathrm{mg} / \mathrm{ml}$ digitonin) allowed antibodies specific for Tim23 (an inner membraneanchored protein) and cyclophilin D (a soluble matrix protein), respectively, to access mitochondria (Figure $6 \mathrm{~b}$ ). In these conditions, a Flag-specific antibody gained access to AIF1 and AIF2 similarly, as soon as the outer mitochondrial membrane was permeabilized (Figures $6 b$ and $c$ ), in concordance with the notion that both proteins expose their flagged C-termini to the intermembrane space.

AIF-deficient cells show reduced abundance of complex I subunits, resulting in a severe respiratory dysfunction. ${ }^{8}$ Accordingly, small interfering RNAs specific for exon 2a or the $3^{\prime}$ UTR of AIF led to a marked reduction in the expression of the $20 \mathrm{kDa}$ complex I subunit (Cl SU20) (Figure 6d) and other complex I subunits (not shown) in U2OS cells (which only express AIF1). The transfection of AIF2 (whose expression is not impeded by either of the two siRNAs) blunted the depletion of $\mathrm{CI}$ SU20 induced by AIF1 knockdown, as much as did the transfection of AIF1 (whose overexpression is abolished by the exon 2a-specific siRNA, yet not affected by the $3^{\prime} U T R$-specific siRNA) (Figure 6d). These results corroborate the hypothesis that both AIF isoforms are localized in the same submitochondrial compartment in which they both sustain the biogenesis or stability of complex I from the respiratory chain. It should be noted that the respiratory and redox functions of AIF are closely linked because redoxdeficient AIF mutants are unable to restore the abundance and function of respiratory chain complex I from AIF knockout cell. $^{26}$ Therefore, it appears plausible that AIF2 has a normal redox function, similar to that of AIF1. Recently, Churbanova et al. $^{27}$ reported a rather low $\mathrm{NAD}(\mathrm{P}) \mathrm{H}$ oxidase activity for a recombinant AIF protein that resembles endogenous AIF more closely than a His-tagged, truncated protein that had been analyzed earlier. ${ }^{7}$ In line with this possibility, we found that the siRNA-mediated depletion of AIF1 and/or AIF2 from SHSY-5Y neuroblastoma cells (which express both AIF1 and AIF2) did not affect cellular $\mathrm{NAD}(\mathrm{P}) \mathrm{H}$ levels (Supplementary Figure 3).

Biochemical differences between AIF1 and AIF2. To detect further possible differences between AIF1 and AIF2, we stably transfected the Flag-tagged versions of both proteins in U2OS cells, obtaining supraphysiological levels of AIF1 and AIF2 (Supplementary Figure 4a). Both Flagtagged AIF1 and AIF2 cDNAs led to the expression of proteins with a similar electrophoretic mobility corresponding to $\sim 65 \mathrm{kDa}$ (Supplementary Figure $4 \mathrm{a}$ ), and similar comigration results were obtained for non-tagged versions of AIF1 and AIF2 (not shown). The overexpression of AIF1 or AIF2 had neither deleterious effect on the proliferation rate (not shown), nor affected the respiratory capacity and control of the cells (Supplementary Figure 4b). However, both AIF isoforms differentially affected the ultrastructure of mitochondria, as detectable by transmission electron microscopy (TEM). AIF1 (but not AIF2) overexpression lead to a rarefaction of cristae, while AIF2 (but not AIF1) overexpression 


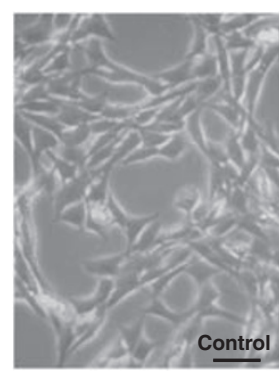

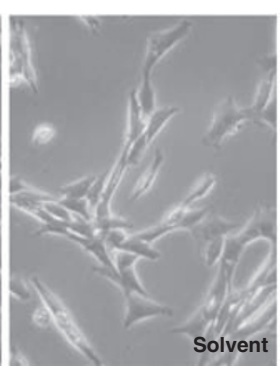

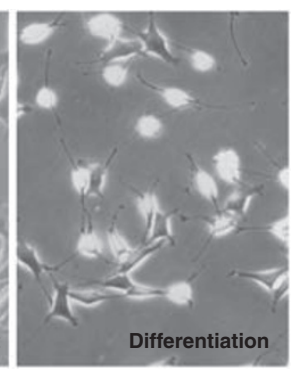

\section{c}

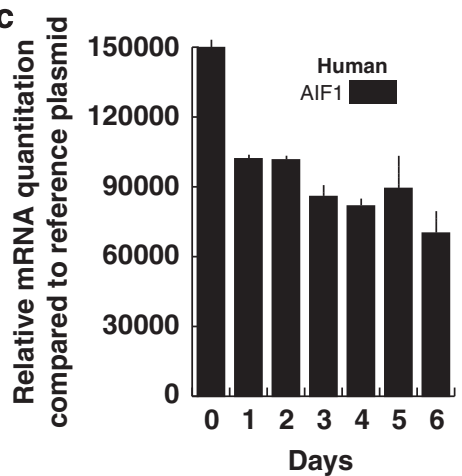

b

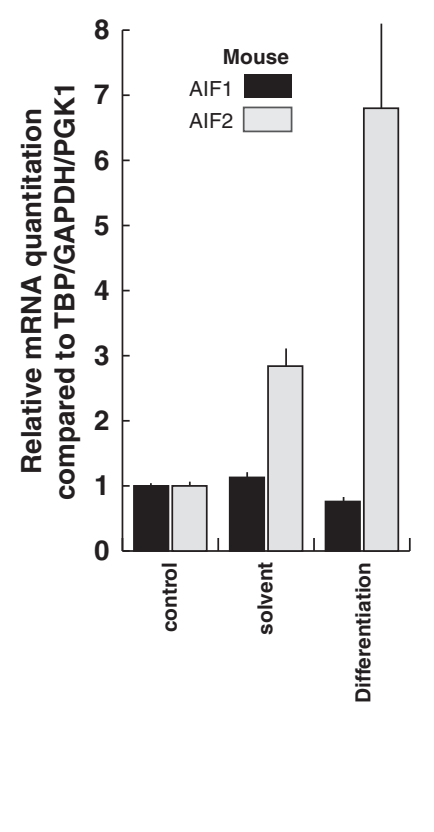

Figure 3 Expression of AIF2 during neuronal differentiation. Murine neural precursor cells (ETNA) were differentiated in vitro into neurons (a) (Bar represents $20 \mu \mathrm{m})$ and AIF1 and AIF2 mRNA expression levels were compared between undifferentiated, solvent-treated and differentiated cells (b). Data represent three independent experiments (mean \pm S.D.). Human mesencephalic cells (LUHMES) were differentiated in vitro into postmitotic dopaminergic neurons and AIF1 and AIF2 mRNA expression levels were compared in RNA samples prepared every day, from day 0 (undifferentiated) up to day 6 after the onset of differentiation (c). Data result from two independent quantifications (means \pm S.D.)

tended to increase the curvature of cristae, which frequently adopted an onion-like shape (Supplementary Figure 4c). Although this result was obtained in conditions in which the two AIF isoforms were overexpressed, it suggested subtle differences in the effect of the insertion of their putative transmembrane domains on mitochondrial membrane structure.

Driven by these results, we analyzed whether AIF1 and AIF2 actually differ in their membrane anchorage, in conditions in which Flag-tagged AIF1 and AIF2 are expressed at physiological levels (lower than endogenous AIF, Figures 7a and b). AIF1 versus AIF2-expressing cells were exposed to increasing concentrations of the non-ionic detergent Igepal CA-630, and the extractability of the two AIF isoforms was measured. While Igepal CA-630 efficiently extracted AIF1 from mitochondria, AIF2 was largely resistant to the detergent and remained bound to the ultracentrifugable pellet. Thus, AIF2 behaves like the inner membrane-anchored CI SU20, while AIF1 behaved like several soluble mitochondrial proteins including cytochrome $c$ (Figure 7a). When, the Igepal CA-630 extraction protocol was replaced by a different one involving sodium carbonate, Flag-tagged AIF1 was again released in conditions of basic $\mathrm{pH}(\mathrm{pH}=10.5)$, whereas Flagtagged AIF2 remained attached to the membrane fraction (Figure 7b). Importantly, in these conditions, endogenous AIF1 (the sole isoform of AIF expressed in HeLa cells) behaved like Flag-tagged AIF1, indicating that membraneanchoring properties of the recombinant AIF was not affected by the Flag tag (Figure 7b). Next, we assessed the effect of the alkylating agent MNNG on the release of AIF1 versus AIF2. Again, it appeared that Flag-tagged AIF1 was readily released into the cytosol of MNNG-treated cells, while Flag-tagged AIF2 remained attached to mitochondria (Figure 7c). In these conditions, endogenous AIF1 co-translocated to the cytosol in the presence of Flag-tagged AIF1. However, endogenous AIF1 was withheld from the cytosol in the presence of Flagtagged AIF2 (Figure 7c).

We conclude that AIF2 is more difficult to be released from mitochondria than AIF1. This difference cannot be explained by the consensus sequence of the cleavage site by calpains and cathepsins, which is the same for both proteins. Rather, the differential release of AIF1 and AIF2 must be linked to other properties of the molecules such as the accessibility of AIF2 to proteases. Thus, the N-terminus of AIF2 might be more profoundly 'buried' in the lipid bilayer of the inner mitochondrial membrane than that of AIF1. In support of this possibility, biochemical studies of AIF1 carried out by Churbanova et al. ${ }^{27}$ suggest that redox-regulated changes in the conformation of AIF that implicate its N-terminal and C-terminal segments might dictate AIF's accessibility to apoptotic proteases and regulate its mitochondrial release in dying cells. In this context, the dimerization of AIF molecules has also been suggested. ${ }^{27,28}$ Indeed, endogenous AIF1 could be detected in the anti-Flag immunoprecipitate of Flagtagged AIF1 or AIF2 (Figure 7d). Thus, the two AIF isoforms can form homo- or heterodimers or higher-order oligomers. Accordingly, we hypothesize that the inhibitory effect of AIF2 on AIF1 release might be explained by a physical interaction 

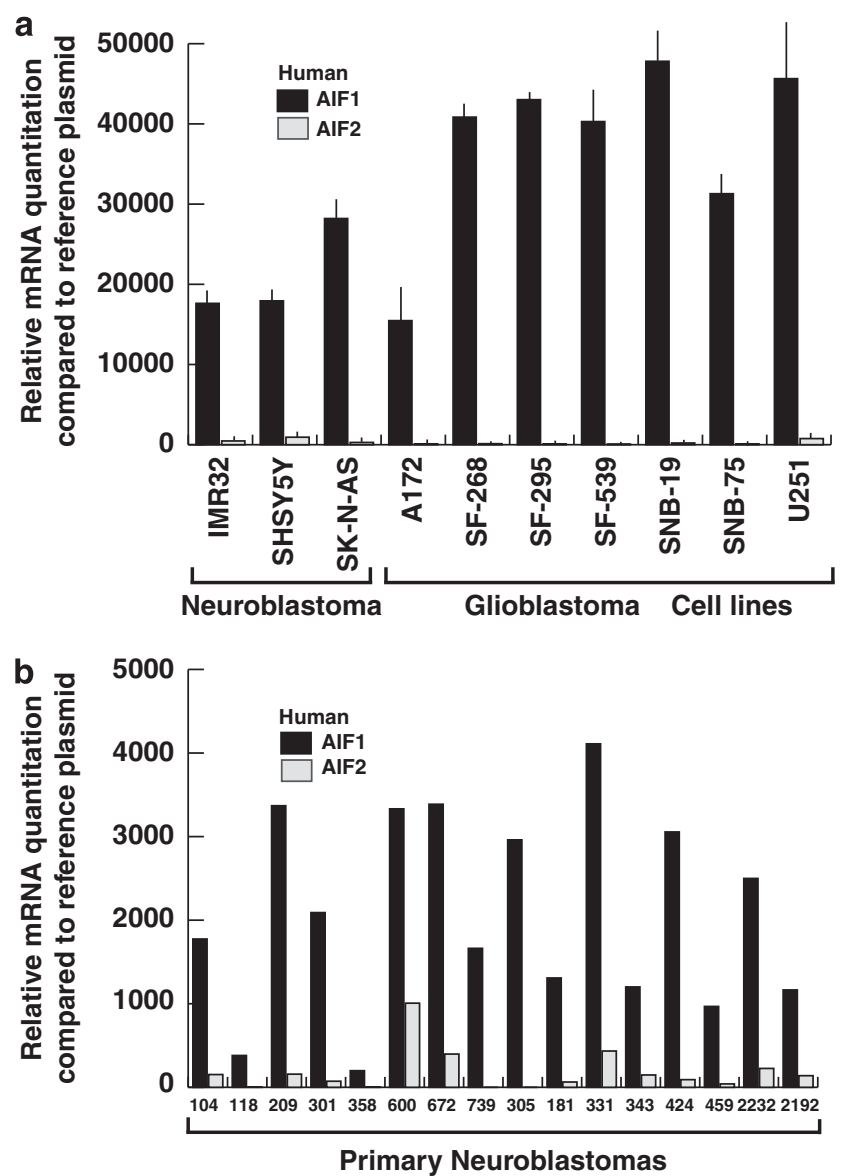

Figure 4 AlF1 and AIF2 mRNA expression profile in cancer cells. Quantitative expression levels of AIF1 and AIF2 mRNAs were measured in human neurablastoma (IMR32, SHSY5Y and SK-N-AS) and glioblastoma (A172 and NCl60 panel: SF-268, SF-295, SF-539, SNB-19, SNB-75 and U251) cell lines (a) as well as primary human neuroblastoma tumors $(\mathbf{b})$. Means \pm S.D. are obtained from two independent experiments

between the two isoforms that makes the mitochondrial release-associated cleavage of AIF1 more difficult to be achieved when AIF2 is present.

Concluding remarks. In this paper, we comparatively analyze two AIF isoforms that solely differ in the utilization of one exon (exon 2) that code for a portion of the AIF Nterminus. Both AIF isoforms, AIF1 and AIF2, are associated with the inner mitochondrial membrane and are similar in their capacity to sustain a normal function of the respiratory chain. Importantly, it appears that the IMSS of AIF1 (IMSSAIF1), which is encoded by exon $2 a$, is cleaved during the import of the AIF precursor into mitochondria, in a highly conserved peptide motif, after residue 54 (in humans AIF1) or residue 53 (in mouse AIF). ${ }^{6,29}$ This implies that only 29 amino acids of the C-terminal half of IMSS-AIF1 are present in the mature mitochondrial AIF1 protein. Importantly, mature mitochondrial AIF2 (as present in brain tissues or obtained by transfection with a cDNA) showed exactly the same electrophoretic mobility as AIF1, suggesting that AIF2 is trimmed during mitochondrial import by a peptidase that cleaves within the exon $2 \mathrm{~b}$-encoded IMSS-AIF2 as well. As a result, the N-terminus of AIF1 and AIF2 are dissimilar in their primary sequence as well as in their hydrophobicity, explaining the relatively loose association of AIF1 (which bears a less hydrophobic $\mathrm{N}$-terminus than AIF2) with the inner mitochondrial membrane. Consequently, AIF2 is more difficult to dissociate from mitochondrial membranes, irrespective of the nature of the desorbing agent (non-ionic detergents, basic $\mathrm{pH}$ or MNNG stimuli), suggesting that AIF2 contributes less efficiently to apoptosis than AIF1.

AIF2 was solely expressed in the developing and adult CNS, as well as in the retina (which contains neurons and, in developmental and anatomical terms, is a CNS derivative). Similarly, AIF2 was present in neuroblastomas but not in any other kind of tumor derived from non-neuronal tissues. Although all CNS cells that express AIF1 also contain AIF2, some cells whose exact nature remains to be identified are positive for AIF2 but not for AIF1. What might be the 'purpose' of CNS-restricted expression of AIF2 in teleological terms? AIF1 and AIF2 share their vital functions with regard to mitochondrial respiration, while AIF2 is more tightly bound to mitochondrial membranes and hence less likely to mediate cell death (which requires the mitochondrial release and nuclear translocation of AIF). If this hypothesis was correct, the utilization of exon $2 b$ (which gives rise to AIF2) might constitute a strategy to avoid AIF-dependent neurotoxicity. As AIF1 and AIF2 can interact with each other and as AIF2 can withhold AIF1 in mitochondria, the putative neuroprotective function of AIF2 would extend to those cells that express both AIF1 and AIF2. For testing this hypothesis, exon 2b-specific knockouts should be generated and evaluated for their putative neurotoxicity alone or upon neuronal injury. As a caveat, it must be mentioned that the expression level of AIF has a profound effect on neuronal differentiation. ${ }^{12}$ Therefore, it remains formally possible that AIF2 (but not AIF1) is intimately linked to CNS development and that the exon $2 \mathrm{~b}$ specific knockout will cause major brain defects. These intriguing hypotheses will be studied in the future.

\section{Materials and Methods}

Antibodies. The following antibodies were used: anti-actin mouse $m A b$ (CHEMICON, Millipore, Billerica, MA, USA, MAB1501); anti-AlF mouse mAb (Santa Cruz Biotechnology, Santa Cruz, CA, USA, Sc13116); anti-Cl SU20 (NDUFB8) mouse mAb (Mitosciences, Euromedex, Mundolsheim, France, MS105); CypD mAb (Mitosciences, MSA04); anti-cytochrome C mouse mAb (Pharmingen, BD Biosciences, Le Pont de Claix, France, 556433); anti-Hsp60 mouse mAb (Stressgen, Tebu, Le Perray en Yvelines, France, SPA-806); antiEndoG rabbit pAb (Cell Signaling, Danvers, MA, USA, 4969); anti-Flag M2 mouse mAb (Sigma, St Louis, MO, USA, F3165); anti-Tim23 mAb (BD Transduction, BD, Le Pont de Claix, France, 611222); polyclonal anti-VDAC rabbit pAb (Cell Signaling, 4866); HRP-conjugated goat anti-mouse (Southern Biotech, 1031-05) and goat anti-rabbit (Southern Biotech, Birmingham, AL, USA, 4010-05); Alexa fluor 488-conjugated goat anti-mouse (Molecular Probes, Invitrogen, Carlsbad, CA, USA, A11029).

Plasmids and siRNA. Recombinant plasmids pCMV-AlF1-3xFlag and pCMVAIF2-3xFlag were constructed by cloning AIF1 and AIF2 open reading frames between EcoR1 and kpn1 sites of the vector pCMV-3xFLAG-CMV-14 (Sigma). The plasmid dsRedmito (Clontech, Ozyme, Saint Quentin Yvelines, France) overexpressing a mitochondrion-targeted dsRed fluorescent protein was used for the tagging of mitochondria in transfected cells. The reference plasmid (Ref-AIF1/ AIF2) used for the relative quantitation of AIF1 and AIF2 expression level by 
a

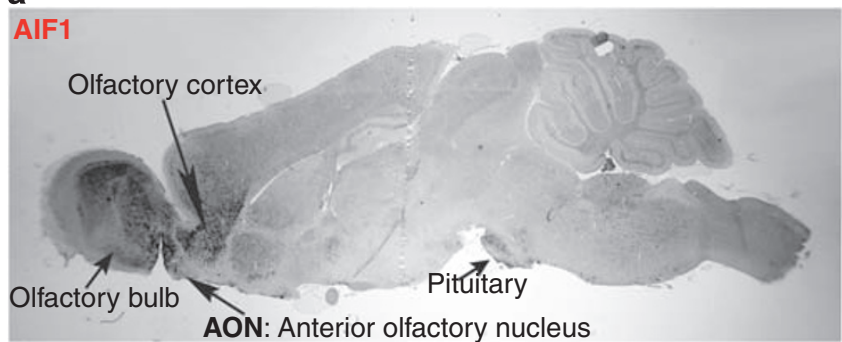

b

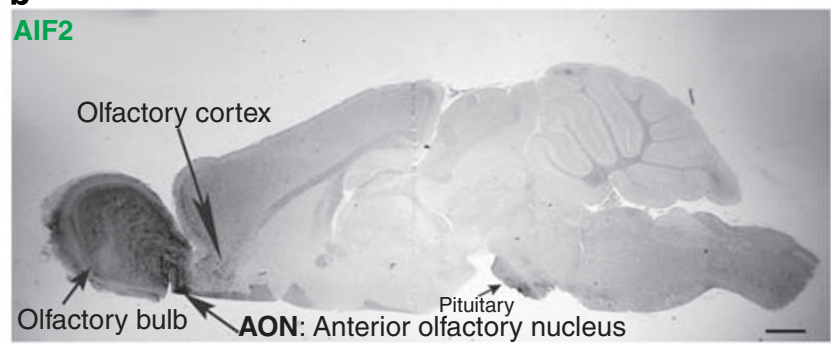

e

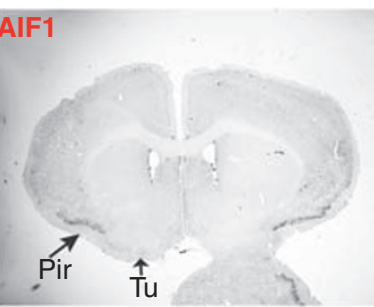

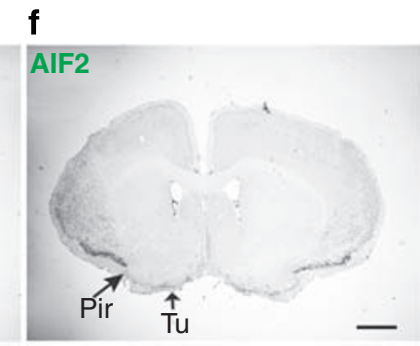

g

AIF1

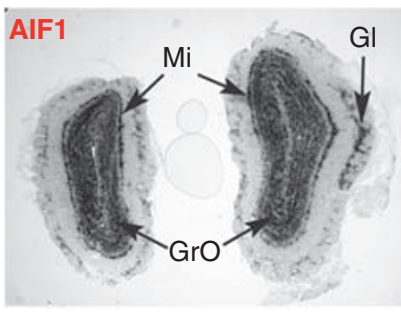

d

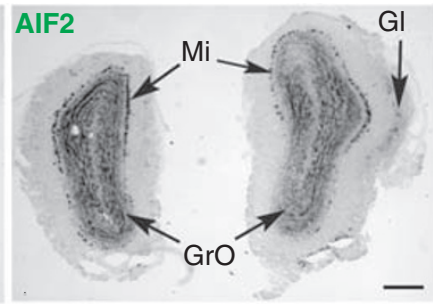

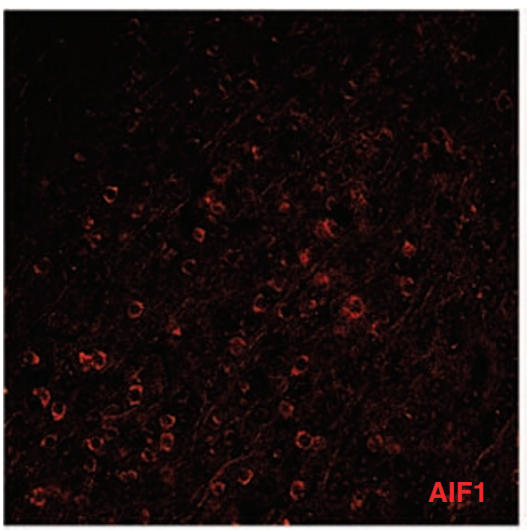

h
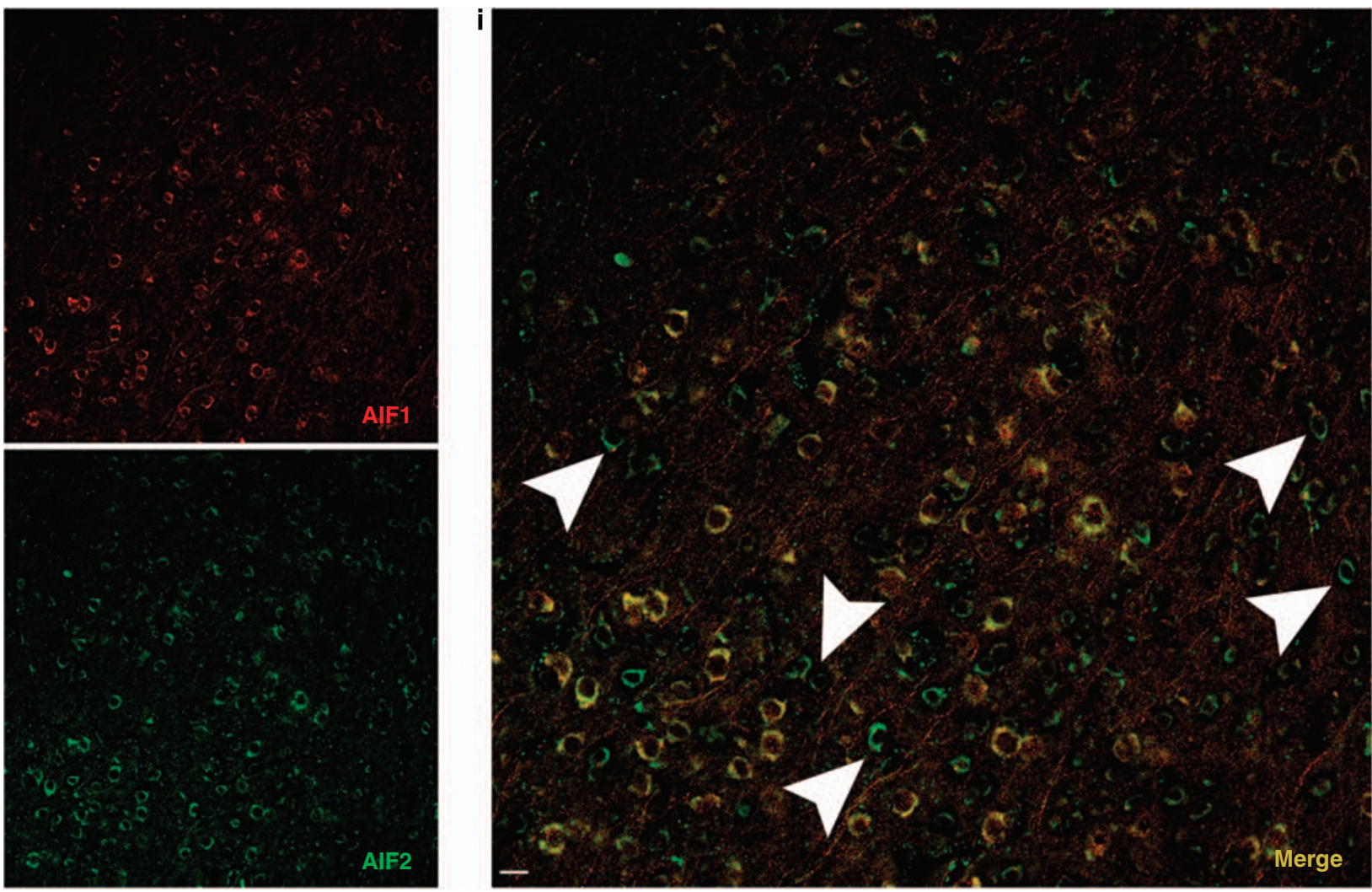

Figure 5 AIF1 and AIF2 mRNA expression pattern in mouse brain. (a-f) Parallel in situ hybridization using respectively AIF1 (a, c, e) and AIF2 (b, d, f) specific probes on brain sagittal sections $(\mathbf{a}, \mathbf{b})$ or the mitral and granular cell layers of the olfactory bulb $(\mathbf{c}, \mathbf{d})$ and cortex $(\mathbf{e}, \mathbf{f})$ on coronal sections $(B a r=1 \mathrm{~mm})$. $(\mathbf{g}-\mathbf{i})$ dual fluorescent in situ hybridization, with AIF1 (red) (g) and AIF2 (green) (h) probes, on the anterior olfactory nucleus (aon) area of brain in sagittal section. The arrows indicate positive cells only for AIF2 in merged images (i) $(B a r=20 \mu \mathrm{m})$. aon, anterior olfactory nucleus; gl, glomerular layer; gro, granular cell layer; mi, mitral cell layer; tu, olfactory tubercle; pir, piriform cortex

standard curve method was constructed by simultaneous head-to-tail ligation of AIF1 (1-438 bp) and AIF2 (1-426 bp) open reading frames and their cloning into Not1 and EcoR1 sites of pGEMTeasy vector (Promega, Charbonniéres-les-Bains, France). For RNA interference experiments the following siRNA sequences were used: Negative control (C ${ }^{-}$) (5́-AUGCAGAACUCCAAGCACGDTDT-3́), AIF-exon 2a (5́-GGGCAAAAUCGAUAAUUCUDTDT-3́), and AIF 3'UTR (5́-GCAGACU UUCUCUGUGUAUDTDT-3́).
Cell culture and transfection. Human Osteosarcoma U2OS cells (ATCC, Manassas, VA, USA, no. HTB-96) as well as cervix carcinoma Hela cells (ATCC no. CCL-2) were cultured at $37^{\circ} \mathrm{C}$ and $5 \% \mathrm{CO}_{2}$ in Dulbecco's modified Eagle's medium (Gibco-Invitrogen, Carlsbad, CA, USA) supplemented with $10 \%$ heat-inactivated fetal bovine serum (FBS) (PAA Laboratories, Pasching, Austria) and 1\% penicillin/ streptomycin. Mouse embryonic fibroblasts (MEF) were cultured in Dulbecco's modified Eagle's medium (Gibco) supplemented with 10\% heat-inactivated FBS 
(PAA), $1 \%$ penicillin/streptomycin (Invitrogen, Carlsbad, CA, USA) and $0.01 \% \beta$ mercaptoethanol. Mouse neural precursor cells (ETNA) were cultured and differentiated in vitro according to Cozzolino et al..$^{30}$ LUHMES (Lund Human Mesencephalic neurons) that were derived by conditional immortalization of female human embryonic ventral mesencephalic cells and subsequent clonal selection, could be differentiated in the presence of GDNF into postmitotic neurons with a robust dopaminergic phenotype. ${ }^{31}$ Briefly, LUHMES were cultured in poly-L-lysine (Sigma) and fibronectin (Sigma) $(50 \mu \mathrm{g} / \mathrm{ml})$-coated culture flasks in proliferation medium, namely DMEM/F12 (Sigma) supplemented with $2 \mathrm{mM}$ L-glutamine (Invitrogen), 1\% N2 supplement, $25 \mu \mathrm{g} / \mathrm{ml}$ bFGF (R\&D Systems $\mathrm{GmbH}$, Wiesbaden-Nordenstadt, Germany) and 1\% penicillin/streptomycin (Invitrogen). Cells were cultured at a density of $2 \times 10^{6}$ and split every 4 or 5 days with proliferation medium changes every 3 days or at $80 \%$ confluence. For differentiation, cells were seeded in six-well plates at a density of 40.000 cells/ $\mathrm{cm}^{2}$ in differentiation medium, namely DMEM/F12, $1 \% \mathrm{~N} 2$ supplement, $1 \mathrm{mg} / \mathrm{ml}$ tetracyclin (Sigma), $49 \mathrm{mg} / \mathrm{ml}$ dibutyryl-cAMP (R\&D Systems) and $5 \mathrm{ng} / \mu \mathrm{l}$ GDNF (R\&D Systems). Every day and up to 6 days after the onset of differentiation, six wells were pooled for RNA extraction.

Plasmid and siRNA transfections were performed using Lipofectamine-2000 reagent (Invitrogen) by following manufacturer's procedure. Pools of stably transfected U2OS cells expressing AIF1-Flag or AIF2-Flag were established by cotransfection with the plasmids described above and the pPuro plasmid (Clontech) followed by selection in the presence of $0.25 \mu \mathrm{g} / \mathrm{ml}$ of puromycin (Clontech) and $0.5 \mathrm{mg} / \mathrm{ml}$ of Geneticin (Invitrogen).

Cell lysates preparation, immunoprecipitation and western blot analyses. For whole extract preparation, cells were harvested, washed three times with ice-cold PBS $\left(8.1 \mathrm{mM} \mathrm{Na}_{2} \mathrm{HPO}_{4}, 135 \mathrm{mM} \mathrm{NaCl}, 1.5 \mathrm{mM} \mathrm{KH}_{2} \mathrm{PO}_{4}, 2.7 \mathrm{mM}\right.$ $\mathrm{KCl}$ ), lysed with $1 \% \mathrm{SDS}$, boiled, sonicated and stored at $-80^{\circ} \mathrm{C}$. Proteins contained in the lysate were quantified (DC protein assay; BioRad Laboratories, Hercules, CA, USA) and resolved directly by SDS-PAGE (NUPAGE; Invitrogen) and then subjected to western blot analyses following manufacturer's instructions (Invitrogen). For immunoprecipitations, cells were lysed for $20 \mathrm{~min}$ at $4{ }^{\circ} \mathrm{C}$ in the following buffer: NetN-120 $(20 \mathrm{mM}$ Tris- $\mathrm{HCl}$ at pH $8.0,120 \mathrm{mM} \mathrm{NaCl}, 1 \mathrm{mM}$ EDTA, $0.5 \%$ Igepal CA 630 ) supplemented with protease (EDTA-free protease inhibitor tablets - Roche Diagnostics Indianapolis, IN, USA) and phosphatase (Phosphatase inhibitor tablets - Roche) inhibitors. After centrifugation $(13000 \times g$ for $10 \mathrm{~min}$ at $4^{\circ} \mathrm{C}$ ), the protein content in the lysates was quantified and used for immunoprecipitations. The following immunoprecipitation procedure was used: first protein G-Sepharose CL-4B beads ( $50 \mu \mathrm{l}$ of a $50 \%$ slurry, GE Healthcare, Piscataway, CA, USA) were coated with $1 \mu \mathrm{g}$ of the indicated antibody, by incubating for $2 \mathrm{~h}$ at $4{ }^{\circ} \mathrm{C}$ in $800 \mu$ l of binding buffer $(20 \mathrm{mM}$ Tris-HCl pH $8.0,120 \mathrm{mM}$ $\mathrm{NaCl}, 1 \mathrm{mM}$ EDTA, $0.5 \%$ Igepal). Then, antibody-coated beads were washed three times in binding buffer and incubated for $2 \mathrm{~h}$ at $4{ }^{\circ} \mathrm{C}$ with $300 \mu \mathrm{g}$ of cell lysate, in a final volume of $800 \mu \mathrm{l}$. Beads were finally washed four times with lysis buffer and coimmunoprecipitated proteins were released by boiling in SB (2\% SDS, $10 \%$ glycerol, $62.5 \mathrm{mM}$ Tris- $\mathrm{HCl} \mathrm{pH} \mathrm{6.8,100} \mathrm{mM} \mathrm{dithiothreitol),} \mathrm{resolved} \mathrm{by} \mathrm{SDS-PAGE} \mathrm{and} \mathrm{then}$ subjected to western blot analysis. For western blot analysis, SDS-PAGE-resolved proteins were transferred onto nitrocellulose membrane (Biorad). Membranes were blocked by incubating with $5 \%$ nonfat milk powder in TBST buffer $(10 \mathrm{mM}$ Tris- $\mathrm{HCl}$ $\mathrm{pH} 8.0,150 \mathrm{mM} \mathrm{NaCl}, 0.05 \%$ Tween 20 ) for $1 \mathrm{~h}$, and then for further $16 \mathrm{~h}$ at $4{ }^{\circ} \mathrm{C}$ with the specified primary antibody diluted in the same incubation mixture supplemented with $0.02 \%$ Na-azide. The membrane was then washed three times in TBST buffer before incubation with a horseradish peroxidase-conjugated secondary antibody. Antibody binding was detected with the $\mathrm{ECL}+$ chemiluminescence detection kit (GE Healthcare).
Indirect immunofluorescence analyses. Cells were fixed on coverslips for 30 min with $4 \%$ PFA $/ 2 \%$ Sucrose $/ 0.19 \%$ Picric acid, and then permeabilized for 5 min with Triton $0.2 \%$ at room temperature. Flag-tagged proteins were detected by incubation with an anti-Flag mAb (1:8000 dilution), followed by Alexa fluor 488conjugated goat anti-mouse. We stained the DNA in the samples with $1 \mu \mathrm{M}$ TOPRO3 (Invitrogen). Samples slides were then mounted using the reagent Fluoromont-G (Southern Biotech) and observed by confocal microscopy with a Leica TSC-SPE confocal microscope equipped with a $\times 63 / 1.15$ Olympus objective and a Leica Aplication Suite (LAS) software (Leica Microsystems, Wetzlar, Germany). For the submitochondrial localization of AIF2, pools of stably transfected U2OS cells overexpressing AIF1-Flag ou AIF2-Flag were first fixed for $30 \mathrm{~min}$ with $4 \%$ PFA $/ 2 \%$ Sucrose $/ 0.19 \%$ Picric acid then permeabilized for 5 min without or with 0.4 and $0.8 \mathrm{mg} / \mathrm{ml}$ of digitonin. Flag-tagged proteins were detected by incubation with an anti-Flag $\mathrm{mAb}(1: 8000$ dilution), followed by a Alexa fluor 488-conjugated goat anti-mouse. Anti-Tim23 mab and CypD mab were used for the detection of mitochondrial inner membrane and matrix respectively. DNA in the samples was stained with TOPRO3. Samples were then mounted and observed with a Leica TSC-SPE confocal microscope.

RNA isolation and gene expression analysis by quantitative realtime PCR (qRT-PCR). Total RNA purified from normal human organs or brain sub-regions were purchased from Clonetech. Total RNA from wild-type (wt) or harlequin $(\mathrm{Hq})$ mutant mice organs were extracted using Precellys homogenizer (Bertin Technologies, Montigny-le-Bretonneux, France) and RNA isolation kit from Qiagen (Courtaboeuf, France). RNA from human and mouse cells in culture were extracted using PARIS kit (Ambion, Applied Biosystems, Applera, Courtaboeuf, France) or 'Total RNA Isolation Kit II' (Macherey Nagel, Hoerdt, France). All RNA samples were then stored at $-80^{\circ} \mathrm{C}$. The quantification of RNA samples was achieved using the Nanodrop ND-1000 Spectrophotometer and the integrity of the RNA was verified using the Agilent 2100 Bioanalyzer with the Eukaryote Total RNA Nano assay. One microgram of total RNA was reverse-transcribed in a $20 \mu \mathrm{l}$ final reaction volume using the high capacity cDNA Reverse Transcription Kit with RNase inhibitor (Applied Biosystems, Applera, Courtaboeuf, France) following the manufacturer's instructions. For the human and mouse AIF1 and AIF2, the following primers and Taqman MGB probes were custom-made by Applied Biosystems: human AIF1 primer (AIF1F): $5^{\prime}$-GGCAAAATCGATAATTCTGTGT TAGTC-3'; human AIF2 primer (AIF2F): $5^{\prime}$-GGAAAGATGGCAGCAACCTAGTG TACT-3'; human AIF commun primer (AIFCr): $5^{\prime}$-CCACCAATTAGCAGGAAAGG AA-3'; human AIF commun probe (AIFcp): $5^{\prime}$-TGTTTCTGTTCTGGTGTCAG-3'; mouse AIF1 primer (AIF1R): $5^{\prime}$-CCATTGCTGGAACAAGTTGC-3'; mouse AIF2 primer (AIF2R): 5'-CTAGGAGATGACACTGCACAA-3'; mouse AIF commun primer (AIFCF): $5^{\prime}$-CGAGCCCGTGGTATTCGA-3'; mouse AIF commun probe (AIFSc): $5^{\prime}$-ACGGTGCGTGGAAG-3'. TaqMan probes were labeled with 6-FAM at the $5^{\prime}$ end and with a nonfluorescent MGB quencher at the $3^{\prime}$ end. Each probe was combined with different forward and reverse primers (see list) for AIF1 or AIF2 quantification. TaqMan gene expression assays for 18S ribosomal RNA (Hs99999901_s1), GAPDH (Mm99999915_g1), PGK1 (Mm00435617_m1), TBP (Mm00446973_m1) and the TaqMan Mouse Endogenous Control Arrays (384-well micro fluidic card containing 16 mouse TaqMan Gene Expression Assays) were from Applied Biosystems. Quantitative PCR reactions were performed using $A B I$ Prism $7900 \mathrm{HT}$ sequence detection system (Applied Biosystems). For microplate experiments, $25 \mathrm{ng}$ of $c D N A$ were used as template for q-PCR reactions with TaqMan Universal Master Mix (Applied Biosystems), $900 \mathrm{nM}$ primers and $200 \mathrm{nM}$ probes. Real-time q-PCR amplifications were carried out $\left(10 \mathrm{~min} 95^{\circ} \mathrm{C}\right.$ followed by 45 cycles of $15 \mathrm{~s} 95^{\circ} \mathrm{C}$ and $1 \mathrm{~min} 60^{\circ} \mathrm{C}$ ). Technical replicates were performed for each biological sample. For the micro fluidic Taqman arrays, $100 \mathrm{ng}$ of cDNA were used per sample-loading ports, each allowing 48 q-PCR reactions using

Figure 6 (a) AIF2 is targeted to mitochondria. HeLa cells transiently co-transfected with the mitochondrial marker dsRed-mito (red fluorescence) and pCMV-AIF1-Flag (top panel) or pCMV-AIF2-Flag (bottom panel) were fixed, immunostained using an anti-Flag antibody (green), counterstained with the DNA-specific dye TOPRO3 for the determination of nuclear area and then observed by confocal microscopy. (b) AIF1 and AIF2 are localized in the same sub-mitochondrial compartment. U20S cells stably overexpressing AIF1-Flag or AIF2-Flag were permeabilized with various concentrations of digitonin and mitochondrial proteins were detected using anti-Flag (AIF1-Flag and AIF2-Flag), anti-Tim23 (inner membrane protein Tim23) and anti-CypD (matrix protein CypD) by confocal microscopy. (c) Histogram of the frequency of cells positively immunostained with each antibody for all permeabilization conditions used in (b). (d) Both AIF1 and AIF2 regulate mitochondrial respiratory chain complex I subunits. Western blot analysis of complex I subunit CI SU20 (NDUFB8). Whole lysates of U20S cells cotransfected with control siRNA, or AIF siRNA (AIF exon 2a or AIF $3^{\prime} U T R$ ) in the presence of empty vector (Flag) or recombinant plasmid pCMV-AIF1-Flag (AIF1-Flag) or pCMV-AIF2-Flag (AIF2-Flag) were subjected to western blot detection of indicated proteins $\operatorname{Bar}=5 \mu \mathrm{m}$ 
manufacturer instructions $\left(10 \mathrm{~min} 94.5^{\circ} \mathrm{C}\right.$ followed by 40 cycles of $30 \mathrm{~s} 97^{\circ} \mathrm{C}$ and $1 \mathrm{~min} 59.7^{\circ} \mathrm{C}$ ). For AIF1 and AIF2 expression quantification in differentiating mouse neural cells, reference genes were selected using the TaqMan Mouse Endogenous Control Arrays. Briefly, 16 housekeeping genes were tested in triplicate for each sample. The most stable genes were selected by analyzing results with GeNorm and Normfinder functions in Genex 4.3.8 (MultiD, Göteborg, Sweden). The geometric mean of the three best housekeeping genes (GAPDH, PGK1 and TBP) was used to normalize gene expression levels of AIF1 and AIF2 for further analysis a

AIF1-Flag

$+$

dsRed mito
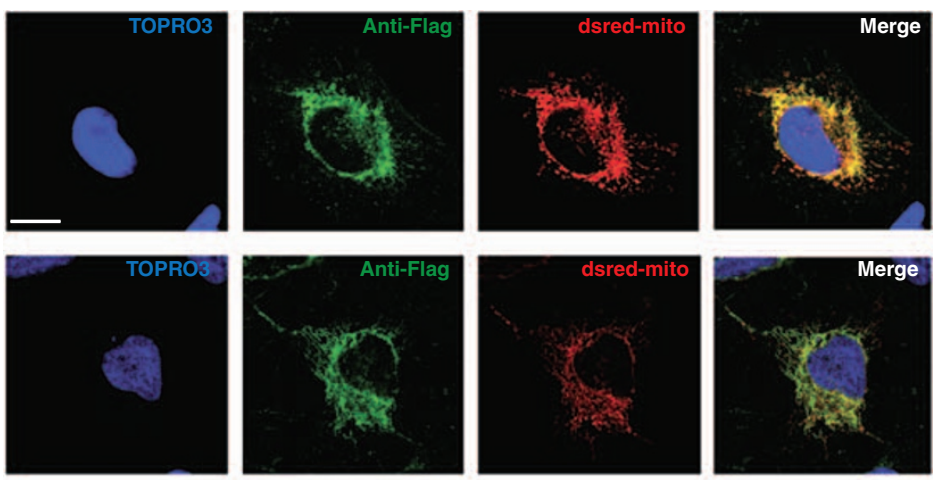

b
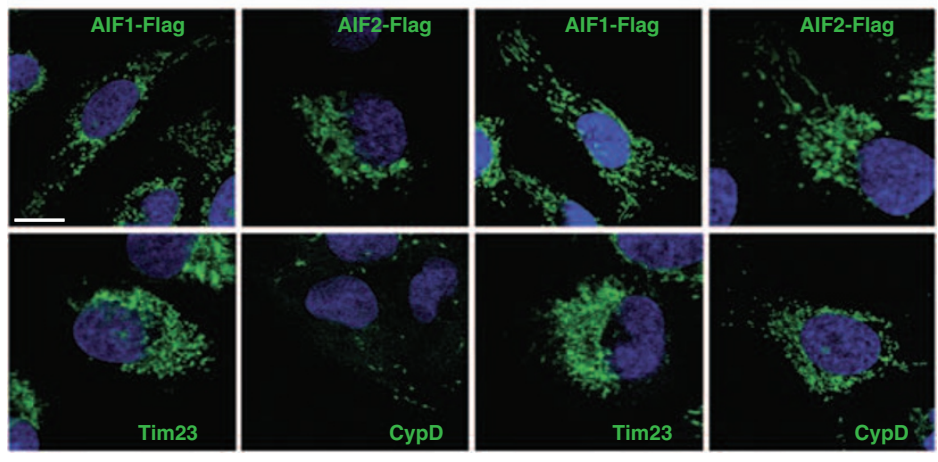

$0.4 \mathrm{mg} / \mathrm{ml}$

$0.8 \mathrm{mg} / \mathrm{ml}$ Digitonin

C

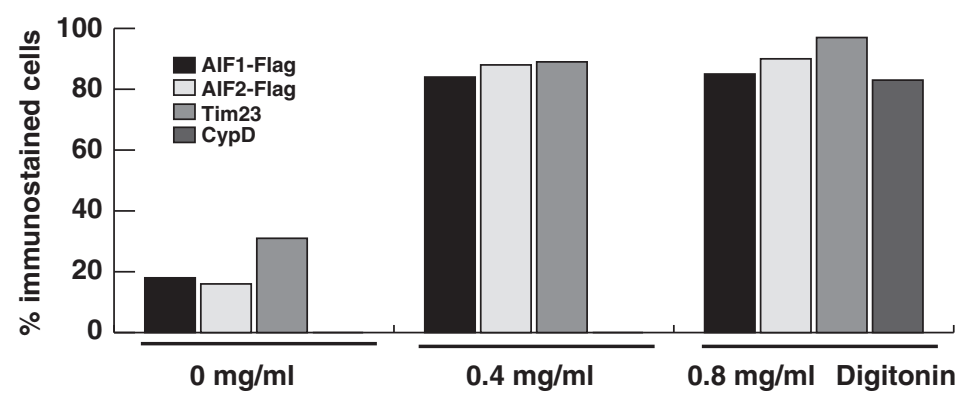

d

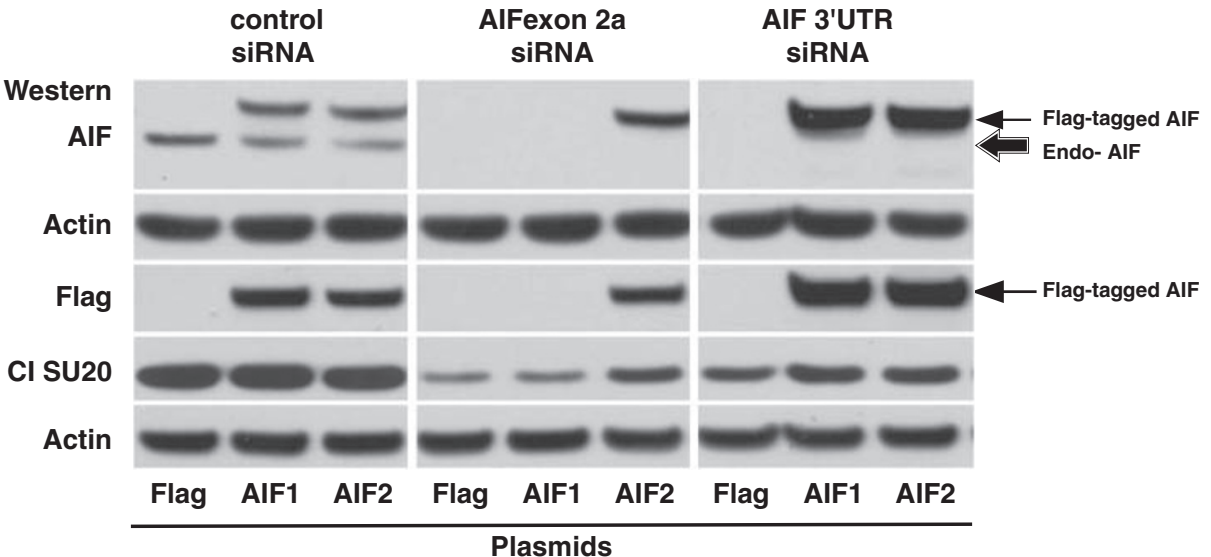


Transfection

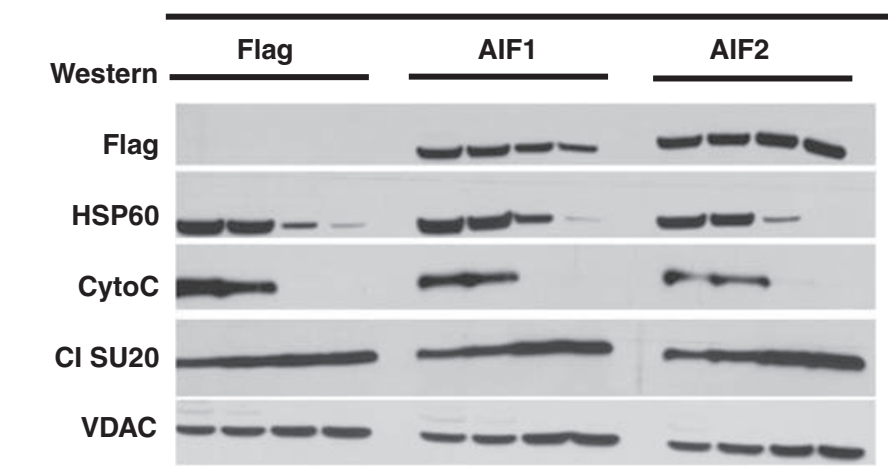

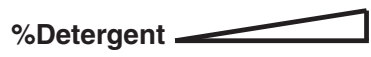

b

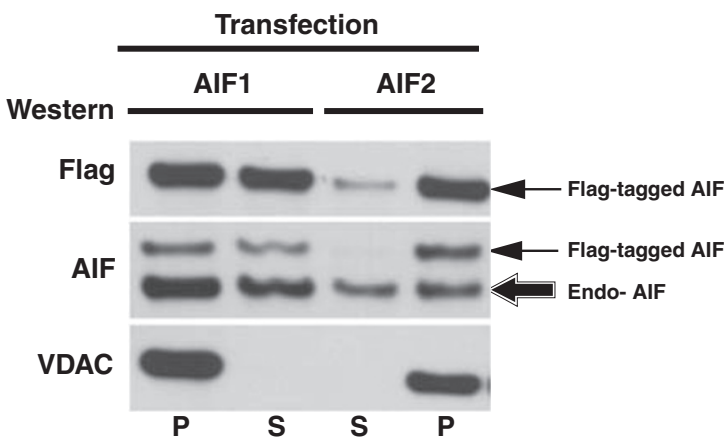

Pellet

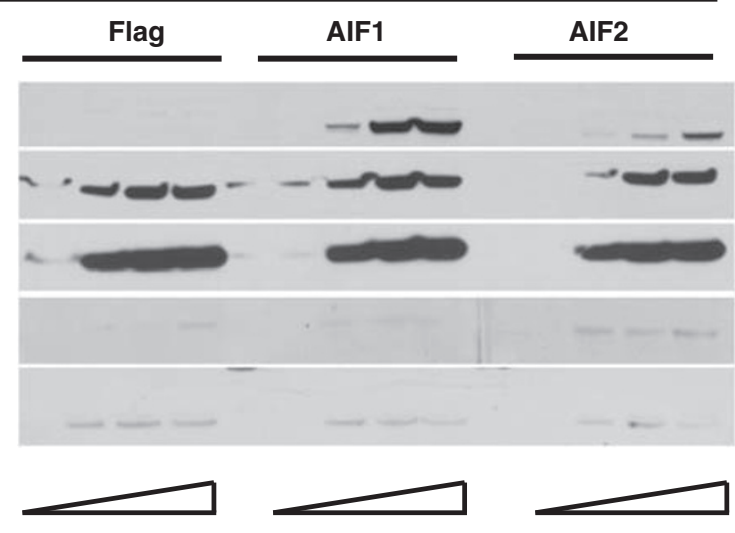

Supernatant

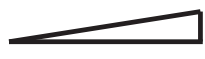

C
Transfection

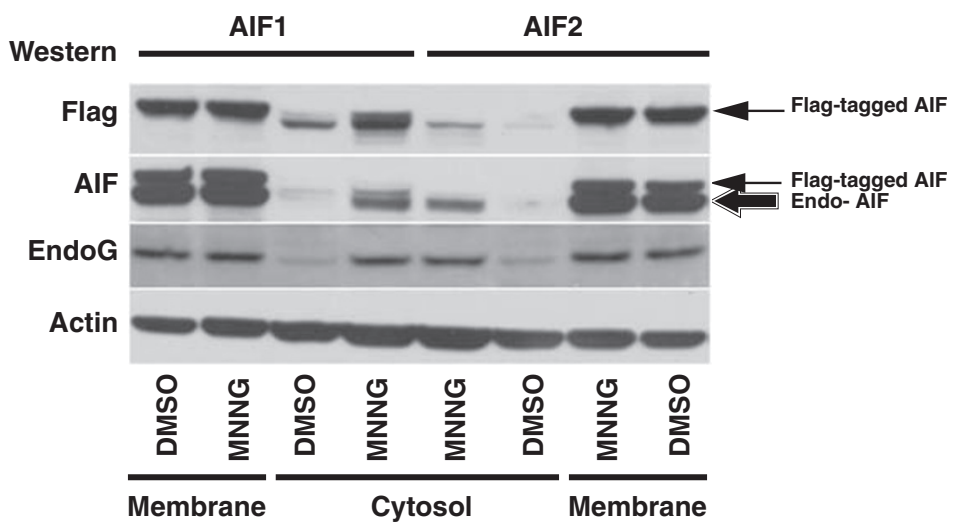

d

Input

Immunoprecipitation

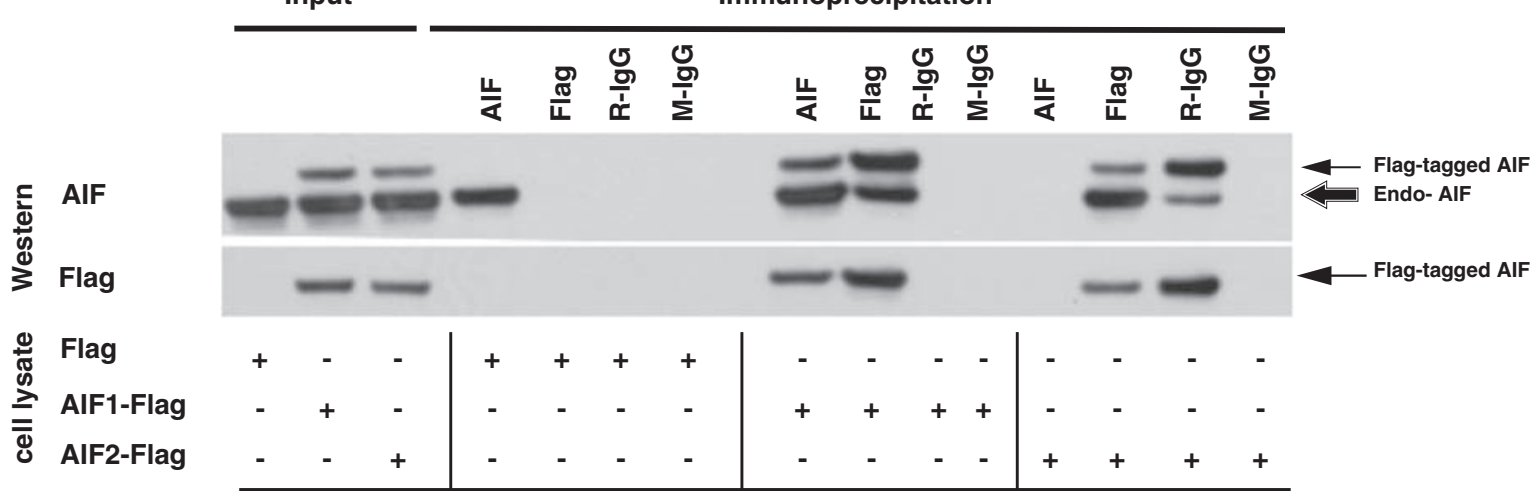

Figure 7 Membrane anchorage capacity of AIF1 and AIF2. (a) Detergent-induced permeabilization of mitochondrial membranes. MEF cells transiently transfected with vector (Flag), pCMV-AIF1-Flag (AIF1-Flag) or pCMV-AIF2-Flag (AIF2-Flag) were incubated without or with increasing concentrations of Igepal CA-630 and then ultracentrifuged to separate the soluble fraction (Supernatant) from the membrane fraction (Pellet). Proteins in both fractions were separated by SDS-PAGE and then analyzed by western blot using antibodies for the indicated proteins. (b) High pH-dependent mitochondrial membrane solubilization. Mitochondria were first purified from HeLa cells overexpressing AIF1-Flag or AlF2-Flag and then incubated with $100 \mathrm{mM} \mathrm{Na-carbonate} \mathrm{(pH} \mathrm{10.5).} \mathrm{After} \mathrm{centrifugation,} \mathrm{supernatant} \mathrm{(S)} \mathrm{as} \mathrm{well} \mathrm{as} \mathrm{pellet} \mathrm{(P)} \mathrm{fractions} \mathrm{were}$ subjected to SDS-PAGE and western blot analyses using the indicated antibodies. (c) Mitochondrial release of AlF1 and AIF2 in MNNG-treated cells. HeLa cells overexpressing AIF1-Flag or AIF2-Flag were treated with DMSO or with the alkylating agent MNNG and then lysed under hypotonic buffer conditions. Cytosolic and membrane fractions obtained after high-speed ultracentrifugation were then subjected to SDS-PAGE and western blot analyses using the indicated antibodies. (d) AIF1 and AIF2 dimerization. HeLa cells transiently transfected with vector (Flag), pCMV-AIF1-Flag (AIF1-Flag) or pCMV-AIF2-Flag (AIF2-Flag) were lysed and submitted to immunoprecipitation using the indicated antibodies. Whole cell lysates (input) as well as co-immunoprecipitated proteins were then resolved by SDS-PAGE and submitted to western blot analyses using the indicated antibodies. Arrows indicate the electrophoretic position of Flag-tagged and endogenous AIF proteins 
of microplate q-PCR experiments with the relative quantification method. For AIF1 and AIF2 expression quantification in human samples, absolute quantification experiment were performed using standard curves obtained by a 10-fold dilution series of a pBlueREF-AIF1 +2 plasmid. Obtained $\mathrm{Ct}$ values for each human sample allowed AIF1 and AIF2 quantifications according to the standard curves using SDS 2.3 software (Applied Biosystems). Determined quantity values were then exploited for further analysis.

Mice manipulation. Wild-type male CD-1 mice of 3-5 months age were used for the in situ hybridization experiments. Animals were anesthetized with Avertin $(0.25 \mathrm{mg} / \mathrm{g}$ body weight, intraperitoneally) and perfused through the heart sequentially, first with PBS (pH 7.4) and then with 4\% paraformaldehyde (PFA). The brains were post-fixed for $24 \mathrm{~h}$ at $4{ }^{\circ} \mathrm{C}$ and cryoprotected with $30 \%$ sucrose/ PBS for $48 \mathrm{~h}$ at $4{ }^{\circ} \mathrm{C}$. Coronal and sagittal cryosections (18-20 $\mu \mathrm{m}$ thick) were obtained by using a cryostat and collected at $-80^{\circ} \mathrm{C}$

In situ hybridization. The CDNA fragments of mouse AIF1 and AIF2 isoforms (NM_012019 and CX_238424) were obtained by RT-PCR from total RNA extracted from adult mouse brain, using the following primers: AIF1 forward $5^{\prime}$-GCAA CTTGTTCCAGCAATGGC- $3^{\prime}$ and reverse $5^{\prime}$-GCACCAGCTCCTATTGTTGAT AAGC-3'; AIF2 forward $5^{\prime}$-GCAGTGTCATCTCCTAGGATC- $3^{\prime}$ and reverse $5^{\prime}$-ATAAATTCCTGCCCCAGTCAC-3'. Different CDNAs were subcloned into the pGemT-easy vector (Promega). The digoxigenin (DIG)- and biotin (BIO)-labeled riboprobes were produced using these plasmids as templates for the in vitro transcription according to the manufacturer's instructions (Roche). Cryosections were fixed in $4 \%$ PFA for $15 \mathrm{~min}$, and then treated with proteinase $\mathrm{K}(20 \mu \mathrm{g} / \mathrm{ml})$ and with $0.2 \%$ glycine for $5 \mathrm{~min}$, respectively. A second fixing with $4 \%$ PFA and $0.2 \%$ glutaraldehyde was followed. The sections were pre-hybridized at $55^{\circ} \mathrm{C}$ for $2 \mathrm{~h}$ in the hybridization buffer $(50 \%$ formamide, $5 \times$ SSC, $10 \%$ blocking reagent, $5 \mathrm{mM}$ EDTA, $0.1 \%$ Tween- $20,0.1 \%$ CHAPS, $0.1 \mathrm{mg} / \mathrm{ml}$ heparin, $1 \mathrm{mg} / \mathrm{ml}$ yeast RNA). The probes labeled with digoxigenin (DIG) or biotin (BIO) were added to the hybridization mix at concentrations of $1 \mathrm{ng} / \mu \mathrm{l}$ and $15 \mathrm{ng} / \mu \mathrm{l}$ in the colorimetric and FISH respectively. The sections were incubated with this solution at $55^{\circ} \mathrm{C}$ over night. The sections were sequentially washed in $2 \times \mathrm{SSC} / 50 \%$ formamide at $50^{\circ} \mathrm{C}$, three times for $30 \mathrm{~min}$, and then in PBS at room temperature. In the colorimetric in situ hybridization, alkaline phosphatase-conjugated anti-DIG antibody and the chromogenic substrate NBT/BCIP were used to detect the DIG-labeled probe, according to the manufacter's protocol (Roche). Images were captured using a Nikon SMZ 800 microscope (Nikon, Tokyo, Japan) equipped with Nikon Coolpix 995 digital camera. In the FISH, the sections were incubated with the anti-DIG antibody conjugated with $\mathrm{Cy} 3$ at concentration of 1:100 (Jackson ImmunoResearch Laboratories, West Grove, PA, USA) for $2 \mathrm{~h}$ at room temperature for the detection of the DIG-labeled probe. After three washes in PBS, the sections were incubated for $30 \mathrm{~min}$ at room temperature with the streptavidin conjugated with Alexa488 at concentration of 1: 100 (Invitrogen) to detect the BIO-labeled probe. There were no apparent signals in control sections with the sense probes. The fluorescence was acquired by using a confocal laser scanning microscope (Leica). The brightness and contrast were adjusted using LAS software (Leica Microsystems).

Differential mitochondrial membrane permeabilization using non-ionic detergent. Mouse embryonic fibroblasts (MEF) transiently overexpressing AIF1-Flag or AIF2-Flag were incubated for $30 \mathrm{~min}$ in buffer $\mathrm{A}$ (20 mM Tris pH8, $120 \mathrm{mM} \mathrm{NaCl}, 1 \mathrm{mM}$ EDTA) or buffer A supplemented with $0.03-0.06-0.1 \%$ of Igepal CA-630 (Sigma). Membrane-bound proteins were separated from soluble proteins by 10 -min centrifugation at $13000 \times g$ and analyzed by western blot.

Mitochondria purification. Cells were incubated for $20 \mathrm{~min}$ in a hypotonic buffer (10 mM Hepes-KOH, pH 7.9; $1.5 \mathrm{mM} \mathrm{MgCl} ; 10 \mathrm{mM} \mathrm{KCl} ; 0.5 \mathrm{mM} \mathrm{DTT})$ supplemented with protease and phosphatase inhibitors (1 tablet/10 $\mathrm{ml}$, Roche). Cells were then lysed with a Dounce homogenizer and centrifuged for $10 \mathrm{~min}$ at $700 \times g$. The corresponding supernatant was transferred to a fresh tube and centrifuged for an additional $15 \mathrm{~min}$ at $12000 \times \mathrm{g}$. The pellet, which corresponded to the mitochondrial fraction, was washed $1 \times$ with the hypotonic buffer and lysed with $1 \%$ SDS.

Mitochondrial membrane solubilization at high $\mathrm{pH}\left(\mathrm{Na}_{2} \mathrm{CO}_{3}\right.$ treatment). Mitochondria were purified from Hela cells transiently overexpressing AIF1-Flag or AIF2-Flag as described above. The post-centrifugation mitochondria-enriched pellet was resuspended and incubated for $20 \mathrm{~min}$ in $100 \mathrm{mM}$ $\mathrm{Na}_{2} \mathrm{CO}_{3}$ ( $\left.\mathrm{pH} 10.5\right)$ and then centrifuged for $1 \mathrm{~h}$ at 65000 r.p.m. Solubilized proteins contained in the supernatant $(\mathrm{S})$ and those still bound to membranes contained in the pellet $(P)$ were lysed $1 \%$ SDS (diluted in $100 \mathrm{mM} \mathrm{Na}_{2} \mathrm{CO}, \mathrm{pH} 10.5$ ) and analyzed by western blotting.

Membrane/Cytosol fraction separation. Hela cells overexpressing AlF1-Flag or AlF2-Flag were incubated for $20 \mathrm{~min}$ in a hypotonic buffer $(10 \mathrm{mM}$ Hepes-KOH pH 7.9; $1.5 \mathrm{mM} \mathrm{MgCl}_{2} ; 10 \mathrm{mM} \mathrm{KCl}$ and $0.5 \mathrm{mM} \mathrm{DTT}$ ) supplemented with protease and phosphatase inhibitors. Cells were then lysed with a Dounce homogenizer and centrifuged for $15 \mathrm{~min}$ at $12000 \times \mathrm{g}$. The pellet, corresponding to the membrane fraction, was washed $1 \times$ with the hypotonic buffer before being lyzed in the same buffer supplemented with $1 \%$ SDS. The supernatant was re-centrifuged at 65000 r.p.m. for $1 \mathrm{~h}$ supplemented with $1 \%$ SDS and used as the cytosolic fraction. To study the mitochondrial release of AIF1 and AIF2 after treatment with MNNG (N-Methyl- $N^{\prime}$-Nitro- $N$-Nitrosoguanidine), Hela cells were treated for $15 \mathrm{~min}$ with $500 \mu \mathrm{M}$ before being re-incubated at $37^{\circ} \mathrm{C}$ with the complete drug-medium for $4 \mathrm{~h}$ and processed for membrane/cytosol fractionation.

Assessment of respiratory chain function. A first spectrophotometric assay was used to successively measure the activity of complex IV (cyanidesensitive cytochrome $c$ oxidase), complex II + III (malonate-sensitive succinate cytochrome $c$ reductase) and complex III (antimycin-sensitive decylubiquinol cytochrome $c$ reductase) in $400 \mu \mathrm{l}$ of medium containing $10 \mathrm{mM} \mathrm{KH}_{2} \mathrm{PO}_{4}, \mathrm{pH} 7.2$ ) and $1 \mathrm{mg} / \mathrm{ml} \mathrm{BSA}{ }^{32}$ This assay measures the redox changes of cytochrome $c$ using two wavelengths $(550-540 \mathrm{~nm})$. A second assay successively measuring the activity of complex I (rotenone-sensitive decylubiquinone NADH reductase) and $\mathrm{V}$ (oligomycin-sensitive ATPase) was performed in $320 \mu$ l of water to which is added $80 \mu$ l of medium consisting of $50 \mathrm{mM}$ Tris ( $\mathrm{pH} 8.0)$, and $5 \mathrm{mg} / \mathrm{ml} \mathrm{BSA}$. It measures NADH oxidation at wavelengths of $340-380 \mathrm{~nm}$ as described earlier. ${ }^{32}$ All measurements were carried out using a Cary 50 spectrophotometer equipped with an 18 -cell holder maintained at $37^{\circ} \mathrm{C}$. All chemicals were of the highest grade from Sigma.

Electron microscopy. Cells were fixed in phosphate buffer $\mathrm{pH} 7.2-3 \%$ glutaraldehyde for $1 \mathrm{~h}$, and post-fixed in $0.1 \mathrm{M}$ cacodylate buffer - $1 \%$ osmium tetroxyde for $2 \mathrm{~h}$. After being rinsed for $5 \mathrm{~min}$ in water and $15 \mathrm{~min}$ in the $0.1 \mathrm{M}$ cacodylate buffer, cells were transferred to $0.2 \mathrm{M}$ cacodylate buffer for $30 \mathrm{~min}$. Cells were washed in $30 \%$ methanol for $10 \mathrm{~min}$, stained in $2 \%$ uranyl acetate in $0.1 \mathrm{M}$ cacodylate buffer $-30 \%$ methanol for $1 \mathrm{~h}$, and washed in $30 \%$ methanol. Cells were then dehydrated in increasing concentrations of ethanol and embedded in Epon 812.

For observations, ultrathin sections were contrasted with $4 \%$ uranyl acetate and lead citrate and examined with a Technail 12 microscope (FEl, Eindhoven, The Netherlands) at $80 \mathrm{Kv}^{33}$

\section{Conflict of interest}

The authors declare no conflict of interest.

Acknowledgements. EH is supported by a fellowship from the Ligue Nationale contre le Cancer. GK is supported by grants from European Union (projects Apo-Sys, ChemoRes, Death-Train), La Ligue Nationale Contre le Cancer (équipe labélisée), Institut National du Cancer (INCa), Agence Nationale de Recherche (ANR). FC is supported by grants from the Telethon Foundation, AIRC and the Italian Ministry of University and Research. PB and PR are supported by grants from European Union (project EumitoCombat), Leducq foundation. GH is supported by the German National Genome Research Network (01GS08136-4). We acknowledge the DTP at $\mathrm{NCl}$ for providing RNA samples for $\mathrm{NCl} 60$ panel of cancer cell lines; Marcel Leist, University of Konstanz, Germany for providing the LUHMES cells; Abdelali Jalil, Didier Métivier and Fulvio Florenzano for help with flow cytometry and confocal microscopy; Eric Jacquet and Imagif (CNRS) Platform for quantitative RT-PCR analyses.

1. Susin SA, Lorenzo HK, Zamzami N, Marzo I, Snow BE, Brothers GM et al. Molecular characterization of mitochondrial apoptosis-inducing factor. Nature 1999; 397: 441-446. 
2. Mate MJ, Ortiz-Lombardia M, Boitel B, Haouz A, Tello D, Susin SA et al. The crystal structure of the mouse apoptosis-inducing factor AIF. Nat Struct Biol 2002; 9: 442-446.

3. Ye H, Cande C, Stephanou NC, Jiang S, Gurbuxani S, Larochette N et al. DNA binding is required for the apoptogenic action of apoptosis inducing factor. Nat Struct Biol 2002; 9 680-684.

4. Modjtahedi N, Giordanetto F, Madeo F, Kroemer G. Apoptosis-inducing factor: vital and lethal. Trends Cell Biol 2006; 16: 264-272.

5. Joza N, Pospisilik JA, Hangen E, Hanada T, Modjtahedi N, Penninger J et al. AlF: not just an apoptosis-inducing factor. Ann NY Acad Sci 2009; 1171: 2-11.

6. Otera H, Ohsakaya S, Nagaura Z, Ishihara N, Mihara K. Export of mitochondrial AIF in response to proapoptotic stimuli depends on processing at the intermembrane space. EMBO J 2005; 24: 1375-1386.

7. Miramar MD, Costantini $P$, Ravagnan L, Saraiva LM, Haouzi D, Brothers $G$ et al $\mathrm{NADH}$ oxidase activity of mitochondrial apoptosis-inducing factor. J Biol Chem 2001; 276: 16391-16398.

8. Vahsen N, Cande $\mathrm{C}$, Briere JJ, Benit $\mathrm{P}$, Joza N, Larochette $\mathrm{N}$ et al. AIF deficiency compromises oxidative phosphorylation. EMBO J 2004; 23: 4679-4689.

9. Klein JA, Longo-Guess CM, Rossmann MP, Seburn KL, Hurd RE, Frankel WN et al. The harlequin mouse mutation downregulates apoptosis-inducing factor. Nature 2002; 419: 367-374.

10. Joza N, Oudit GY, Brown D, Benit P, Kassiri Z, Vahsen N et al. Muscle-specific loss of apoptosis-inducing factor leads to mitochondrial dysfunction, skeletal muscle atrophy, and dilated cardiomyopathy. Mol Cell Biol 2005; 25: 10261-10272.

11. Cheung EC, Joza N, Steenaart NA, McClellan KA, Neuspiel M, McNamara S et al. Dissociating the dual roles of apoptosis-inducing factor in maintaining mitochondrial structure and apoptosis. EMBO J 2006; 25: 4061-4073.

12. Ishimura R, Martin GR, Ackerman SL. Loss of apoptosis-inducing factor results in celltype-specific neurogenesis defects. J Neurosci 2008; 28: 4938-4948.

13. van Empel VP, Bertrand AT, van der Nagel R, Kostin S, Doevendans PA, Crijns HJ et al. Downregulation of apoptosis-inducing factor in harlequin mutant mice sensitizes the myocardium to oxidative stress-related cell death and pressure overload-induced decompensation. Circ Res 2005; 96: e92-e101.

14. Benit $P$, Goncalves $S$, Dassa EP, Briere JJ, Rustin $P$. The variability of the harlequin mouse phenotype resembles that of human mitochondrial-complex I-deficiency syndromes. PLOS ONE 2008; 3: e3208.

15. Vahsen N, Cande C, Dupaigne $\mathrm{P}$, Giordanetto $\mathrm{F}$, Kroemer RT, Herker $\mathrm{E}$ et al. Physical interaction of apoptosis-inducing factor with DNA and RNA. Oncogene 2006; 25 1763-1774.

16. Cande C, Vahsen N, Kouranti I, Schmitt E, Daugas E, Spahr C et al. AIF and cyclophilin A cooperate in apoptosis-associated chromatinolysis. Oncogene 2004; 23 1514-1521.

17. Gogvadze V, Orrenius S, Zhivotovsky B. Multiple pathways of cytochrome $c$ release from mitochondria in apoptosis. Biochim Biophys Acta 2006; 1757 639-647.
18. Norberg E, Gogvadze V, Ott M, Horn M, Uhlen P, Orrenius S et al. An increase in intracellular $\mathrm{Ca} 2+$ is required for the activation of mitochondrial calpain to release AIF during cell death. Cell Death Differ 2008; 15: 1857-1864.

19. Gurbuxani S, Schmitt E, Cande C, Parcellier A, Hammann A, Daugas E et al. Heat shock protein 70 binding inhibits the nuclear import of apoptosis-inducing factor. Oncogene 2003; 22: 6669-6678.

20. Zhu C, Wang X, Deinum J, Huang Z, Gao J, Modjtahedi N et al. Cyclophilin A participates in the nuclear translocation of apoptosis-inducing factor in neurons after cerebral hypoxiaischemia. J Exp Med 2007; 204: 1741-1748.

21. Galluzzi L, Blomgren K, Kroemer G. Mitochondrial membrane permeabilization in neuronal injury. Nat Rev Neurosci 2009; 10: 481-494.

22. Schulthess FT, Katz S, Ardestani A, Kawahira H, Georgia S, Bosco D et al. Deletion of the mitochondrial flavoprotein apoptosis inducing factor (AIF) induces beta-cell apoptosis and impairs beta-cell mass. PLOS ONE 2009; 4: e4394.

23. Loeffler M, Daugas E, Susin SA, Zamzami N, Metivier D, Nieminen AL et al. Dominant cell death induction by extramitochondrially targeted apoptosis-inducing factor. FASEB J 2001; 15: 758-767.

24. Delettre C, Yuste VJ, Moubarak RS, Bras M, Lesbordes-Brion JC, Petres S et al. AIFsh, a novel apoptosis-inducing factor (AIF) pro-apoptotic isoform with potential pathological relevance in human cancer. J Biol Chem 2006; 281: 6413-6427.

25. Delettre C, Yuste VJ, Moubarak RS, Bras M, Robert N, Susin SA. Identification and characterization of AIFsh2, a mitochondrial apoptosis-inducing factor (AIF) isoform with NADH oxidase activity. J Biol Chem 2006; 281: 18507-18518.

26. Urbano A, Lakshmanan U, Choo PH, Kwan JC, Ng PY, Guo K et al. AlF suppresses chemical stress-induced apoptosis and maintains the transformed state of tumor cells. EMBO J 2005; 24: 2815-2826.

27. Churbanova IY, Sevrioukova IF. Redox-dependent changes in molecular properties of mitochondrial apoptosis-inducing factor. J Biol Chem 2008; 283: 5622-5631.

28. Sevrioukova IF. Redox-linked conformational dynamics in apoptosis-inducing factor. J Mol Biol 2009; 390: 924-938.

29. Yuste VJ, Moubarak RS, Delettre C, Bras M, Sancho P, Robert N et al. Cysteine protease inhibition prevents mitochondrial apoptosis-inducing factor (AIF) release. Cell Death Differ 2005; 12: 1445-1448.

30. Cozzolino M, Ferraro E, Ferri A, Rigamonti D, Quondamatteo F, Ding H et al. Apoptosome inactivation rescues proneural and neural cells from neurodegeneration. Cell Death Differ 2004; 11: 1179-1191.

31. Lotharius J, Falsig J, van Beek J, Payne S, Dringen R, Brundin P et al. Progressive degeneration of human mesencephalic neuron-derived cells triggered by dopaminedependent oxidative stress is dependent on the mixed-lineage kinase pathway. $J \mathrm{Neurosci}$ 2005; 25: 6329-6342.

32. Benit P, Goncalves S, Philippe Dassa E, Briere JJ, Martin G, Rustin P. Three spectrophotometric assays for the measurement of the five respiratory chain complexes in minuscule biological samples. Clin Chim Acta 2006; 374: 81-86.

33. Luft JH. Improvements in epoxy resin embedding methods. J Biophys Biochem Cytol 1961 9: 409-414. 